Invited talk presented at the 9 th Intermational Conference on High Energy Physics and Nuclear Structure (9-ICOHEPANS), Versailies, France (July 6-10, 1981 ).

ORO 5126-145

U. of Md. PT \#82-002

CONF-810747--3

DYNAMICS OF HADRON-NUCLEUS INTERACTIONS

Scephen $J$. Wallace

Department of Physics and Astronomy

University of Maryland, College Park, Maryland 20742 U.S.A.

July 1981

ASU S-7C ERO 2126

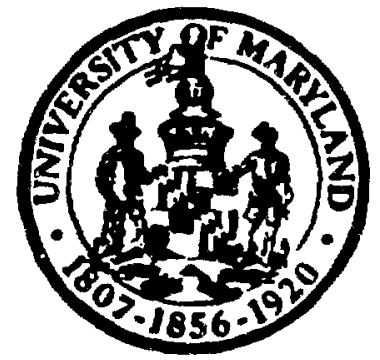

UNIVERSITY OF MARYLAND

DEPARTMENT OF PHYSICS AND ASTRONOMY

COLLEGE PARK, MARYLAND

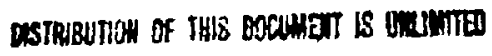




\title{
DYNAMICS OF HADRON-NUCLEUS INTERACTIONS
}

\author{
Stephen J. WALLACE
}

Department of Physics and Astronomy, University of Maryland College Park, Maryland 20742 U.S.A.

Abstract: Recent progress in diffraction theory shows that proton-nucleus scattering at nonforward angles is dominated by the interference of waves from two or more "bright spots". Analytic formulas based on asymptotic theorles of diffraction yield valuable new insights into the scattering and these formulas can be readily extended to illuminate the role of dynamical ingredients, f.e., the nucleon-nucleon amplitudes. The governing parameters of the diffraction and some direct connections between the observed cross secthons and the inp: $t$ dynamics are reviewed. New information regarding the nucleon-nucleon parameters hased on recent phase shift analyses show some systematic differences from the "effective" NN amplitudes which produce fits to proton-nucleus diffraction data. Recent progress in understanding the role of $:$-isobars in proton-nucleus dynamics is reviewed.

\section{Introduction}

Beautiful diffraction patterns are produced by elastically scattering fast hadrons from nuclei. In recent years, experimental programs at Gatchina and Saclay:-17) using $1 \mathrm{GeV}$ proton beams and at Los Alamos ${ }^{8-30}$ ) using a $0.8 \mathrm{GeV}$ proton beam have concentrated on the accumulation of accurately detailed protonaucleus diffraction data. The experiments include some exceptional examples of high resolution in both energy and angle together with high statistical accuracy. Figure 1 displays some recent diffraction angular distributions from the High Resolution Spectrometer at Los $A_{1 a m o s}{ }^{28}$ ). The characteristic oscillations arise from interference of waves diffracting from an equivalent absorptive disk and there is an interfercmetric sensitivity to the ratius. A second characteristic feature of the patterns is the approximate exponential decrease with $q$ for nonforward angles as indicated by the dotted lines in fig. 1 .

Fig. 1 Differential cross section data points $(\cdot)$ and KMT optical potential fits for elastic scattering of $800 \mathrm{MeV}$ protons by Ca isotopes $^{28}$ ).

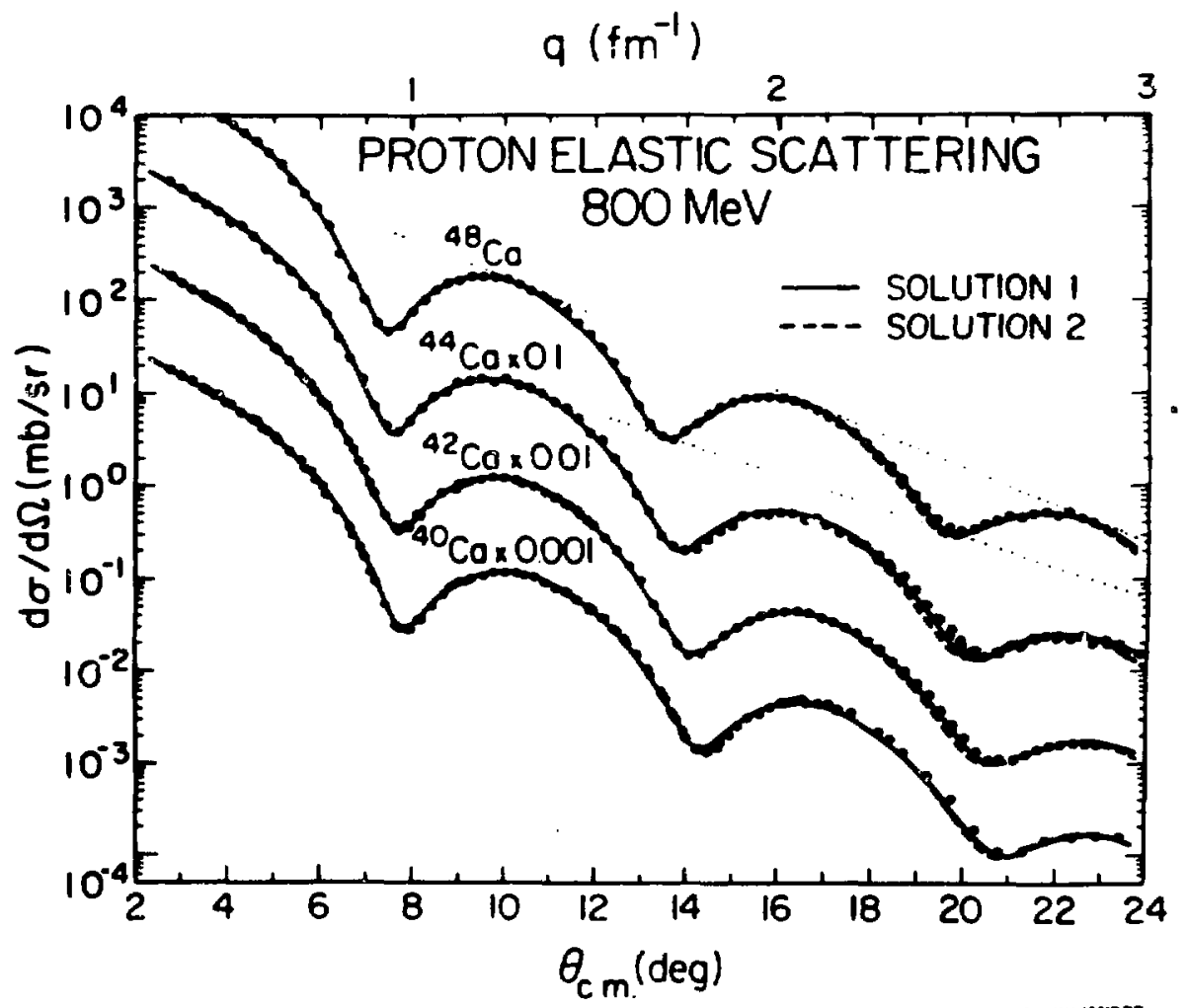


Polarization data also display some very simple features as shown in fig. 2, particularly when plotted versus $-9 \mathrm{~A}^{1 / 3}$ to line up the diffractive oscillations.

Fig. 2 Analyzing power data for $800 \mathrm{MeV}$ proton-nucleus elastic scattering.

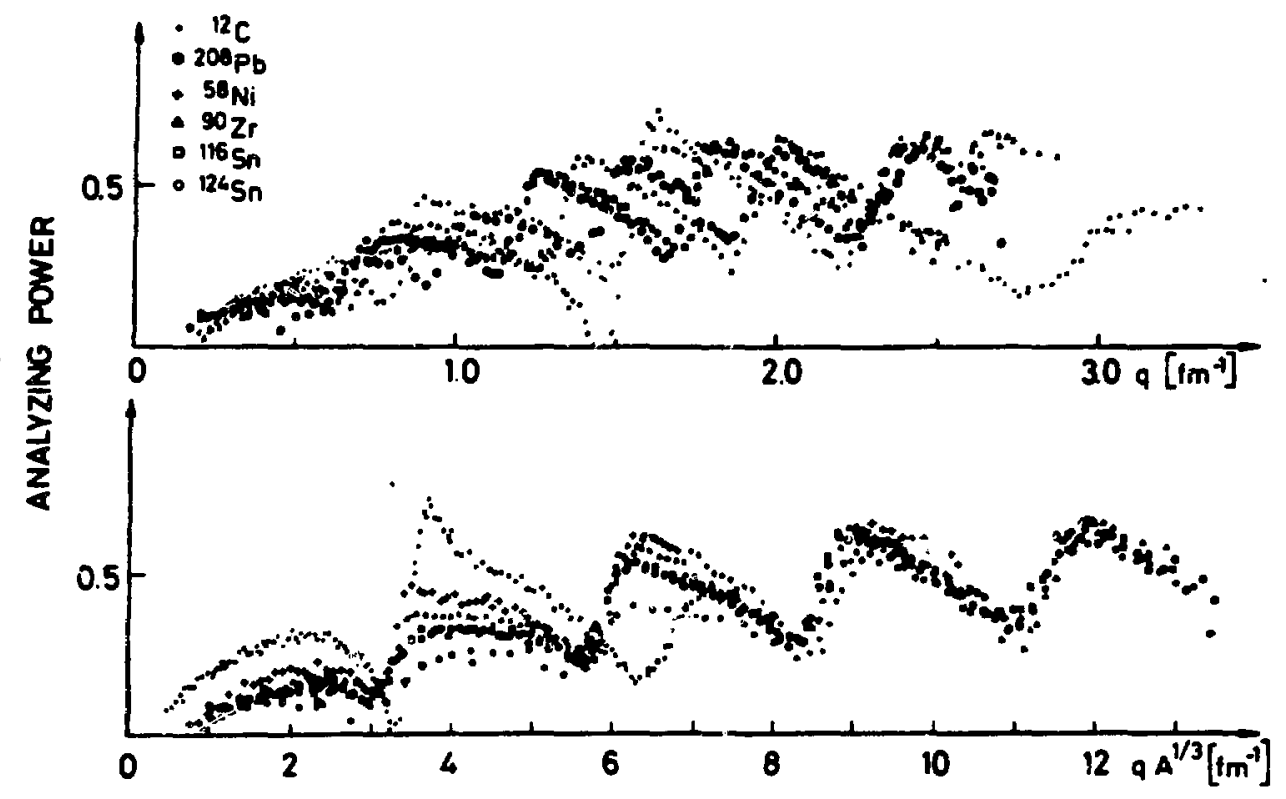

Similar experimental resulis are now energing from Los Alamos and Vancouver near $0.5 \mathrm{GeV}$ and lower energies. Figure 3 shows $0.4 \mathrm{GeV}$ proton scattering from $208_{\mathrm{fb}}{ }^{31}$ ) Because the cross section data are shown as the ratio to Rutherford scattering, the characteristic exponential fall-off appears as a curve in this plot. In the foreseeable future, the diffraction scattering will be mapped out from $0.2 \mathrm{GeV}$ (Indiana) through $1 \mathrm{GeV}$.

Fig. 3 Elastic cross section and analyzing power data for $400 \mathrm{MeV}$ proton scattering by $\mathrm{Pb} .{ }^{31}$ )

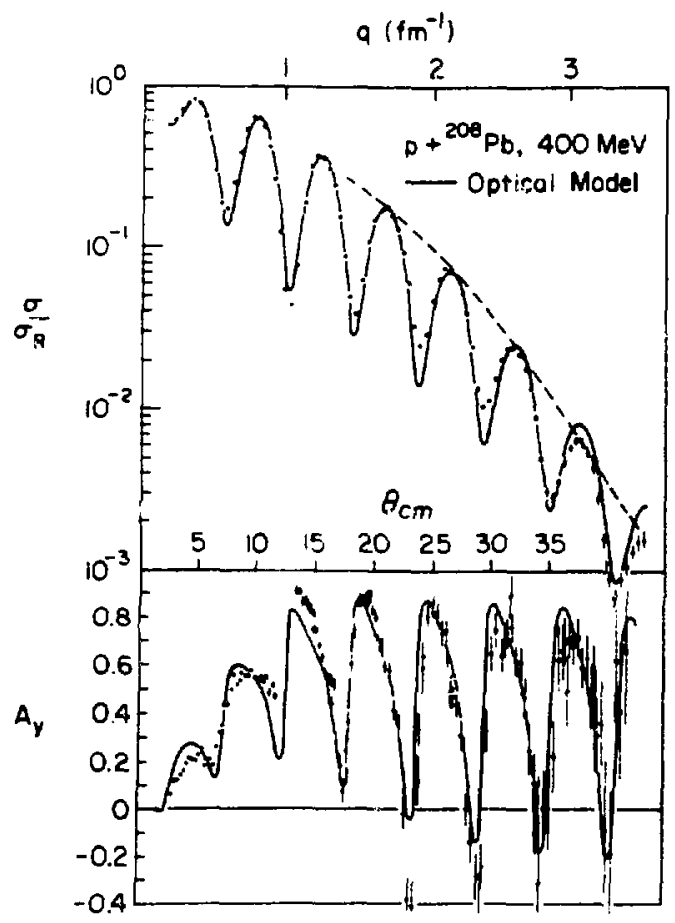


At the higher energies, it has been established by many calculations that the proton data can be successfully interpreted using the simplest multiple scattering theories, i.e., Glauber's multiple diffraction theory ${ }^{32-56}$ ) or the Kerman, McManus, Thaler optical potentlal approach ${ }^{57-72}$ ). Generally these calculations incorporate simple assumptions about the dynamics, $1 . e .$, the NN amplitudes, because accurate NN iniormition has not been available. One of the reasons for some confidence in the muitiple scattering calculations is that a number of second-order effects, e.g. , Pauli correlations, center-of-mass correlations, noneikonal effects, the influence of double spin flip terms in the $N$ interaction and the infiuence of the magnetic spin flip interaction have all been studied to some degree. Generally there are sig.lificant but minor effects due to second-order corrections. For quantitative accuracy one needs to include them in theoretical analyses.

Recent reviews by Alkhazov, Belostotsky and Vorobyov ${ }^{73}$ ), Whitten ${ }^{74}$ ), Ray ${ }^{75}$ ), Igo $^{76}$ ), Thomas ${ }^{77}$, and Wallace ${ }^{78}$ ) have discussed many aspects of proton-nucleus scattering. In this article the emphasis is on dynamics, However, dynamical issues are concealed behind the predouinant and basically simple diffraction structure for $A Z 12$ nuclei. Thus a primary issue has become the separation of diffraction and dynamics and there are some genulne bright spots which can be reported in this area (sec. 2).

Progress in NN amplitude determinations is discussed in sec. 3 and a sumary of recent work on the role of $\Delta$-isobars in proton-nucleus interactions is given in sec. 4.

\section{Bright spots}

Recent developments of asymptotic expressions for large momentum transfer have added considerable insight into proton-nucleus diffraction. In a very interesting paper. Amado, Dedonder and Lenz ${ }^{79}$ ) considered the diffraction produced by protons scattering from a Woods-Saxon density using the eikonal approximation. They showed that a pair of saddle points at complex values of the impact parameter account for most of the features of diffraction by nuclei for $q \gtrsim 1 \mathrm{fm}^{-1}$.

Unpublished work by Glauber and Bleszynski ${ }^{80}$ ) illustrates that saddle points are present for density forms other than the Woods-Saxon and that there can be multiple saddle points when strong Coulomb effects are present, for example, in heavy ion scattering. However, high energy proton-nucleus diffraction seems to involve just two saddle points. There

are in effect two slits rather firmly lodged in the nuclear surface as indicated in fig. 4. Typically at $800 \mathrm{MeV}$, for angles of $10^{\circ}$ to $50^{\circ}$, the scattering is dominated by the rays emerging from these bright spots. The nucleus is rather similar to a two-slit interferometer!

Fig. 4 Upper sketch indicates protons scattering near the edge of the nucleus. Lower sketch indirates slit-shaped bright spots which domilate large $q$ elastic diffraction. 
Amado, Dedonder and Lenz employ the standard Fourier-Bessel impact parameter representation for the diffraction integral;

$$
f(q)=\frac{k}{i} \int_{0}^{\infty} d b \quad b \quad J_{0}(q b)\left[s_{F}(b)-1\right] \text {. }
$$

The impact-parameter amplitude $S_{F}(b)$ contains all the information about nuclaar geometry, nucleon-nucleon amplitudes and electromagnetic effects. Glauber and Bleszynski employ a two-dimensional form where the $x$-axis is parallel to momentum transfer $\vec{q}$. The impact parameter amplitude $S_{F}(\vec{b})$ need not be rotationally invariant in this case and the diffraction integral over the $\vec{b}$-plane is done in two steps:

$$
f(q)=\frac{k}{2 \pi i} \int_{-\infty}^{\infty} d x e^{i q x} S(x), \quad s(x)=\int_{-\infty}^{\infty} d y S_{F}(x, y)
$$

In either approach, $S_{F}$ is rather smoothly varying and the oscillations of $J_{0}(q b)$ or $i e^{i q x}$ produce dramatic cancellations when $q R$ is 1 arge, where $R$ is the nuclear radius. Figure 5 illustrates the situation for the diffraction integral ( 1 ).

Direct numerical evaluation of eq. (1) is a standard task in diffraction calculations, however this approach provides lit.tle insight regarding how the nuclear geometry and the dynamics (e.g., the NN amplitudes) affect the observed diffraction. Similar loss of insight holds for the partial wave calculations based on the optical model. The situation is dramatically improved by deforming the contour of integration into the complex impact parameter plane since there are points where rapid variations of $S_{F}(b)$ can match the oscillations due to the Bessel function. This technique has a long tradition in scattering theor $\mathrm{y}^{81}$ ) analysis

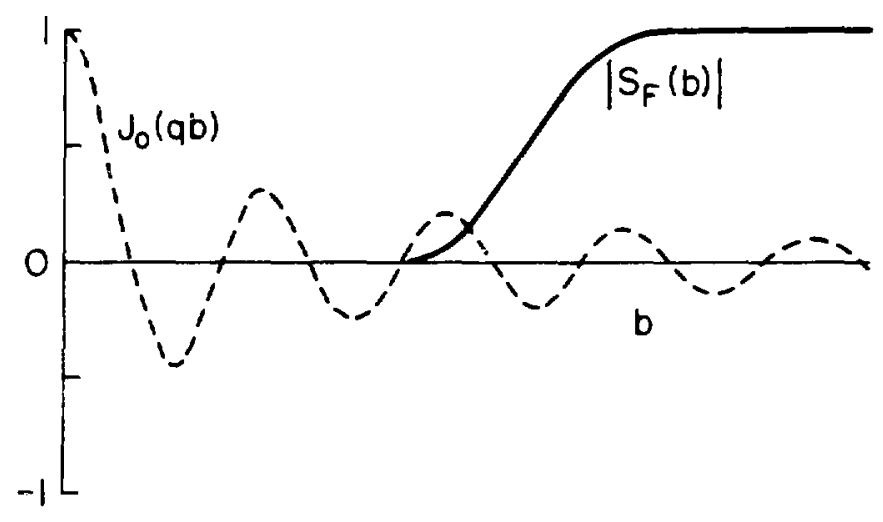
but has only recently been applied to the high energy proton scattering. Convergence requires that one first separate the Bessel function into two parts due to the asymptotic behavior for large values of $q \mathrm{~b}$ :

$$
J_{0}(q b) \sim \frac{1}{2}\left(\frac{2}{\pi q D}-\right)^{1 / 2}\left[e^{i(q b-\pi / 4)}+e^{-i(q b-\pi / 4)}\right] \text {, }
$$

one of which is exponentially damped in the upper-half b-plane while the other has that property in the lower-half b-plane. Thus, two amplitudes are of interest at large $q$ :

$$
f^{( \pm)}(q)=\frac{k}{2 i} \int_{0}^{\infty} d b b\left(\frac{2}{\pi q b}\right)^{1 / 2} e^{ \pm i(q b-\pi / 4)} s_{F}(b) \text {. }
$$

Since $q \neq 0$, the 1 part of eq. (1) produces no contribution and therefore

$$
f(q)=f^{(+)}(q)+f^{(-)}(q) \text {. }
$$

The observed cross section involves the interference

$$
\sigma(q)=\sigma^{(+)}(q)+\sigma^{(-)}+2\left[\sigma^{(+)}(q) \sigma^{(-)}(q)\right]^{1 / 2} \cos [\phi(q)] \text {, }
$$

where $\sigma^{( \pm)}(q)=\left|f^{( \pm)}(q)\right|^{2}$ and $\phi(q)$ is the phase difference between $f^{(+)}$and $f^{(-)}$. The observation of Amado, Dedonder and Lenz is that a pair of complex stationary phase points, 1.e., saddle points, dominate $f^{(+)}$and $f^{(-)}$. The integration con- 
tour for $\mathrm{f}^{(+)}$is deformed into the upper-half $b$-plane to pass through the saddle point $b_{+}$nearest to the real axis and simllarly the contour for $f(-)$ is deformed into the lower-half b-plane to pass through the nearest saddle point (b-) as 1llustrated in fig. 6 . The saddle points are defined by the stationary phase requirement, i.e., the first derivative of the phase of the integrand must vanish;

$\left.\frac{d}{d b}\left[\frac{1}{2} \ln b+\ln S_{F}(b)\right]\right|_{b=b \pm} \pm i q=0$.

Consequently the solutions $b_{ \pm}$ of this relation depend on $q$ and the geometrical and dynamical parameters which control the transmission function $S_{F}(b)$. A second requirement which is needed to optimize the saddle point contributions is that the contours of integration must be chosen

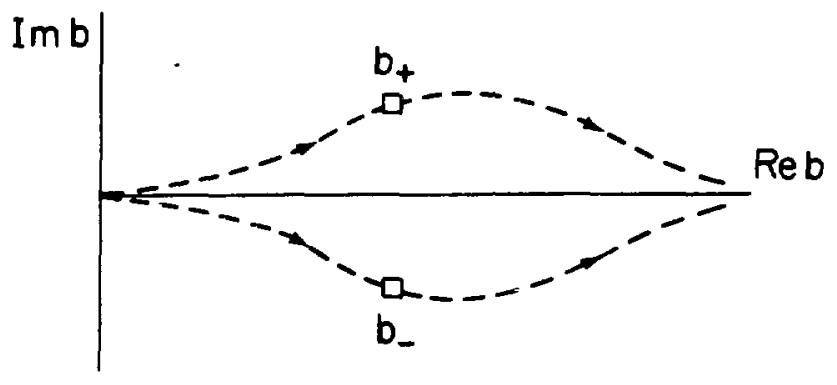
to pass through the points $b_{ \pm}$in the "steepest descent" direction which is specified by the phase of a complex length $\ell_{ \pm}$. Near a saddle point the integrand in eq. (4) takes the form

$$
\left(\frac{2 b_{ \pm}}{\pi q}\right) 1 / 2 e^{i\left(q b_{ \pm}-\pi / 4\right)} s_{F}\left(b_{ \pm}\right) e^{-\pi\left(b-b_{ \pm}\right)^{2} / l_{ \pm}^{2}}
$$

where the only b-dependence retained is the Gaussian factor which governs the phase variation. Complex length parameters $\ell_{ \pm}$are defined in terms of the second derivative of the complex phase at the saddle point and consequently they also depend on $q$ and the other parameters.

The saddle point contributions to the scattering amplitudes are easily evaluated by carrying out the b-integration with the result,

$$
f^{( \pm)}(q)=\frac{k}{2 i} \ell_{ \pm} S_{F}\left(b_{ \pm}\right)\left(\frac{2 b_{ \pm}}{\pi q}\right) 1 / 2 e^{ \pm i\left[q \operatorname{Re} b_{ \pm}-\pi / 4\right]} e^{-q\left|\operatorname{Im} b_{ \pm}\right|} .
$$

Thus once the saddle points $b_{ \pm}$are located, the bright spot amplitudes are calculated from eq. (9) and the interference pattern is calculated from eq. (6). The presence of dominant sadule points is actually quite common for proton-nucleus scattering and does not rely on specific analytic forms for $S_{F}(b)$. The asymptotic methods therefore provide a very useful simplification of the diffraction problem but without any loss of dynamical information. This is a decisive advantage over many other approaches.

The general features noted in the introduction Indicate that $\operatorname{Re}\left(b_{ \pm}\right)$is approximately the nuclear radius and that $\mathrm{Im} b_{ \pm}$is approximately constant and is related to the diffusivity of the optical density. Exactly these features were shown to prevail by Amado, Dedonder and Lenz for a minimal model based on a WoodsSaxon nuclear density,

$$
\rho_{m}(r)=\frac{\rho_{0}}{1+\exp ((r-c) / a)},
$$

where $c$ is the half-density radius and $a$ is the diffusivity.

In the eikonal approximation one has

$$
\begin{aligned}
S_{F}(b) & =e^{-\gamma t(b)} \\
t(b) & =\int_{-\infty}^{\infty} d z \rho_{m}\left(\sqrt{z^{2}+b^{2}}\right)
\end{aligned}
$$


where

$$
\gamma=\frac{\sigma}{2}(1-10)
$$

is a constant involving the isospin average NN cross section, $\sigma$, and a real to Imaginary ratio, $\cap$. The Coulomb interaction is not included at this level but that is easily remedied. Analytic solutions for the saddle points in this example are approximately given by

$$
b_{ \pm}=c \pm 1 \pi a-\frac{1}{2}\left[\frac{\alpha^{2}(c \pm i \pi a)}{q^{2}}\right]^{1 / 3} \exp \left( \pm i \frac{2 \pi}{3}\right)
$$

where $\alpha=2 \pi \gamma$ a $\rho_{0}$ is a dimensionless constant. In the special case of the WoodsSaxon density, eq. (11) shows that the dominant saddle points tend to fixed values $c \pm 1 \pi a$ as $q \rightarrow \infty$. This is due to the existence of poles of the density at $r=c \pm i \pi a$. However, the existence of saddie points does not require such specific analytic properties of the density. The point is that the optical thickness function may be defined as a Fourier transform of a real form factor $F(q)$ miltiplied by the scalar $N N$ amplitude $A(q)$, as follows:

$$
\gamma t(b) \rightarrow \bar{A}(b)=2 \int_{0}^{\infty} d q q J_{0}(q b) F(q) A(q) \text {. }
$$

From eq. (3) one sees that, for complex b, there is an exponentially growing contribution which leads to arbicrary values of $t^{\prime}(b)$. Thus solutions to the saddle point condition ( 7 ) can generally be found.

When the analysis is carried through and Coulomb effects are inserted, the following asymptotic form for the diffraction cross section emerges ${ }^{79}$ ):

$$
\sigma(q)=\left|A_{0}\right|^{2} \frac{e^{\varepsilon(q c)^{1 / 3}}}{q^{8 / 3}} e^{-2 \pi q a}\left\{e^{2 \psi}+e^{-2 \psi}+2 \cos (2 q c+\phi)\right\}
$$

where $A_{0}$ is a constant and $\psi$ and $\phi$ are slowly varying functions of $q$. This expression displays both the diffraction oscillations with period $\approx \mathrm{c} / \pi$ that arise directly frou the nuclear half-density radius $c$ and an exponential decay factor $e^{-2 \pi q a}$. The factor in brackets oscillates between $4 \cosh ^{2} \psi$ and $4 \sinh ^{2} \psi$ envelopes, thus the function $\psi$ describes filling of diffraction minima and it involves the Coulomb parameter $\left(n=Z e^{2} / \mathrm{Kv}\right)$ and the ratio $(0)$ of the real to imaginary parts of the forward NN amplitude as follows:

$$
\psi \simeq \pi[-2 \pi a / c]+\rho\left[\frac{1}{2}(q c)^{1 / 3}(\pi a / \lambda)^{2 / 3}-.51(\pi a)^{3 / 2} /\left(\lambda c^{1 / 2}\right)\right] .
$$

In this expression, $\lambda=\left(\sigma \rho_{0}\right)^{-1}$ is the proton's mean free path. As $\psi$ increases slowly with (qc) ${ }^{1 / 3}$, the envelope of oscillations narrows as is illustrated by the convergence of the dotted lines in $\mathrm{fig}$. 1 . Recent large $q$ data 29 ) on ${ }^{12} \mathrm{C}$ provide an example where the oscillations essentially disappear. These features cañ be understood in terms of one of the bright spot amplitudes becoming dimmer than the other by the factor $e^{-\psi}$ due to a differing optical path length through the nuclear surface. The function $\phi$ provides a slow variation of the pariod of oscillation due to the difference of optical paths of the two interfering rays:

$$
\phi \simeq \frac{3}{2}(q c)^{1 / 3}(\pi a / \lambda)^{2 / 3}+5 \pi / 3-.51(\pi a)^{3 / 2} /\left(\lambda c^{1 / 2}\right) .
$$

Figure 7 shows a comparison of the saddle point cross section formula with accurate numerical integration of the diffraction integral. The reproduction of the numerical calculations is very good except for the most forward angles.

Some care is needed before concluding that the exponential fall-of of the cross section necessarily implies the expone:atial fall-off of the density. Glauber and Bleszynski ( $f$ ig. 8 ) have shown that even for a Gaussian density there is an approximate exponentlal fall-off of the cross section for a finite range of $q$. It is the signature of saddle points which move slowly with $q$, the movement being controlled by $q^{-2 / 3}$ for the Woods-Saxon case of eq. (11) and by $\ln (q)$ for the Gaussian case. 


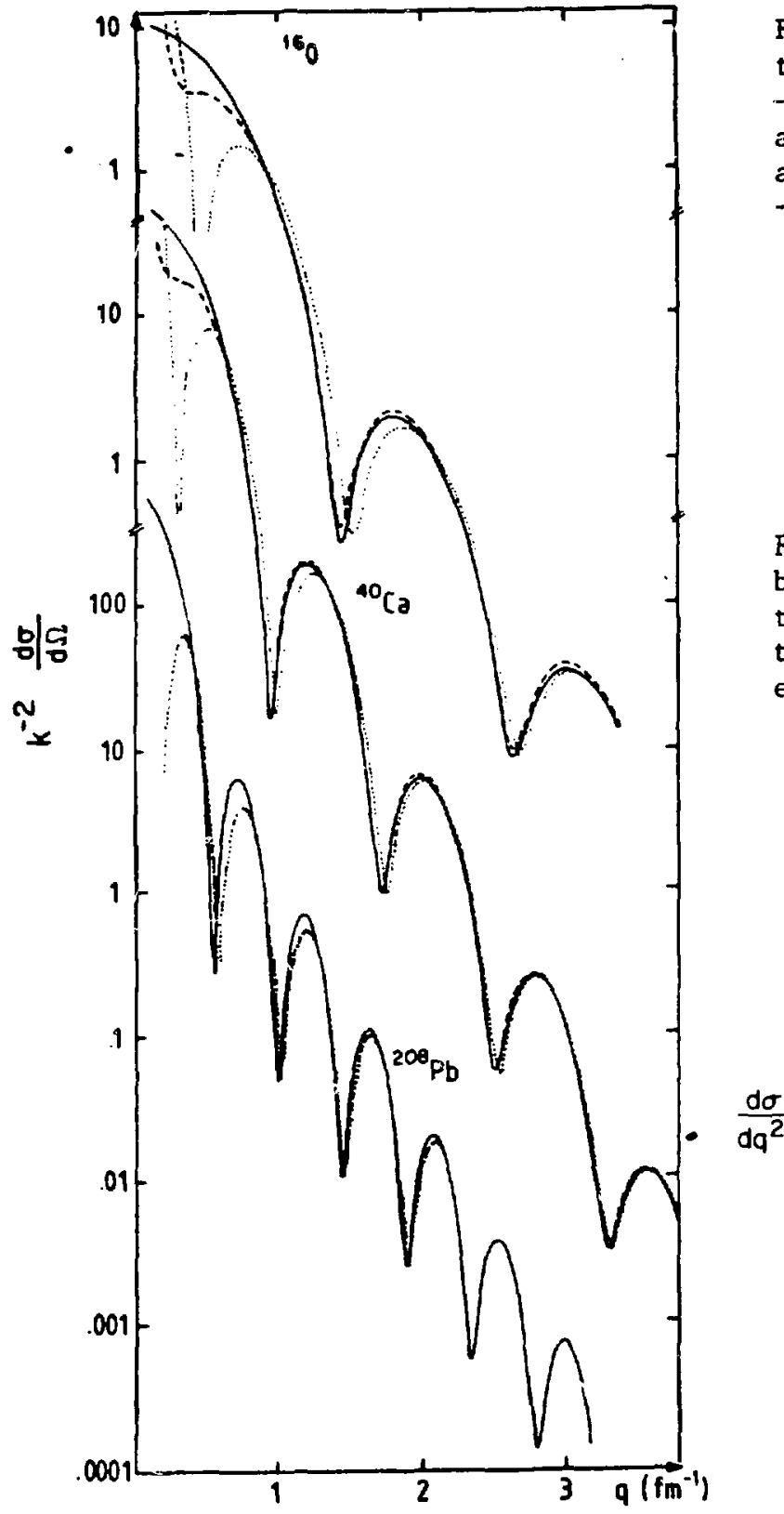

Fig. 7 Numerical result ${ }^{79}$ ) based on the diffraction integral of eq. (1). - is compared to the simplest asymptotic formula result $\cdots \cdots$ and to an improved asymptotic formula result - - - - .

Fig. 8 Differential cross section ${ }^{80}$ ) based on a Gaussian thickness function $t(b)$ as in eq. (10b) but calculated in the asymptotic approximation to eqs, (2).

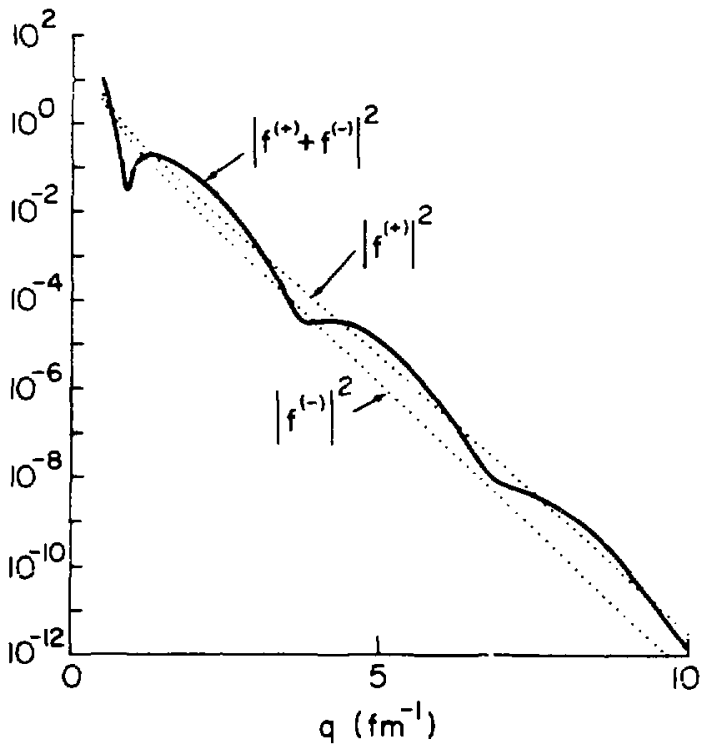

Although derivation of the asymptotic formulas (13)-(15) involves quite a few approximations, the results are reasonably accurate and they provide valuable insight to the diffraction for $q \gtrsim 1 \mathrm{fm}^{-1}$. For the first time one has a direct connection between the cross section and the nuclear geometry and $\mathrm{NN}$ amplitude parameters. Alternative methods must be employed for small $q$ a d there has been recent work relevant to that regime also. Germond and Johnson ${ }^{32}$ ), and in a more general approach, Frahn ${ }^{83}$ ), have considered analytic approaches to diffraction using nonasymptotic methods. There is considerable overlap in the angular range of validity of the asymptotic and nonasymptotic methods. However, analytic results in nonasymptotic diffraction theory generally rely on specific analytic forms for the impact parameter amplitude $S_{F}(b) .84,85$ ) The importance and flexibility of the asymptotic methods described above lies in the fact that the dynamical information need not be lost by forcing $S_{F}(b)$ to take some convenient 
analytic form. This point is fllustrated by the following example which shows how the range of the NN amplitude may be incorporated.

As is well known in Glauber theor ${ }^{86}$ ), the optlcal density involves the range $B_{A}$ of the $N N$ amplitude which has been taken to be zero in the approximation of eq. (10). Employing a Gaussian NN amplitude $A(q)=A_{0} e^{-B_{A} q^{2}}$ in eq. (12), and assuming a Woods-Saxon density as in eq. (10), the main effect of the NN range parameter is to cause a larger root-mean-square radius for the optical density than for the nuclear density via the relation $r_{\mathrm{opt}}^{2}=r_{\text {nuc }}^{2}+6 \beta_{\mathrm{A}}$. An approximate way to incorporate this effect in the spirit of analytic approximations is to ask for the new Woods-Saxon parameters copt and a for for the optical density which preserve the volume integral and incorporate the shift of rms radius. Generaily the effects are small and they can be approximated to first order in $\beta_{A}$ by the following relations:

$$
\begin{aligned}
& c_{\text {opt }}=c-2 \beta_{A} /\left(c+\frac{7}{15} \frac{\pi^{2} a^{2}}{c}\right) \\
& a_{\text {opt }}^{2}=a^{2}-6 B_{A} / \pi^{2}
\end{aligned}
$$

Figure 9 compares the optical density for ${ }^{40} \mathrm{Ca}$ with the nuclear density.

Fig. 9 Solid line shows a Woods-Saxon nuclear density for ${ }^{40} \mathrm{Ca}$ and dashed line shows the equivalent WoodsSaxon optical density based on eqs. (16) for $B_{A}=0.2 \mathrm{fm}^{2}$.

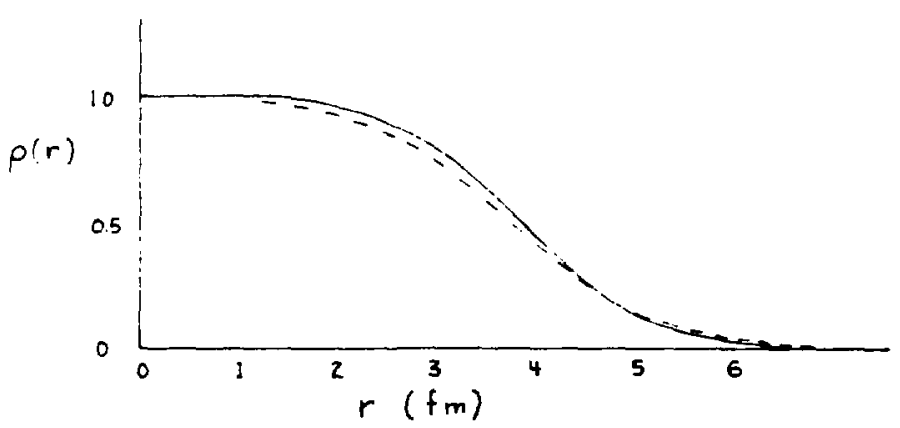

It is a simple matter to extend the analytic results (13)-(15) to include the influence of the $\beta_{A}$ parameter by using $c_{\text {opt }}$ and $a_{\text {opt }}$ in place of the nuclear parameter $c$ and a of eq. (10a). This seemingly trivial incorporation of the dynamical parameter $B_{A}$ into the analytic relations would not be possible unless there was a direct connection between the cross section and the optical density. The small shifts between the optical and nuclear parameters illustrated in fig. 9 are, in fact, important. A principal source of uncertainty in the extraction of neutron radii from high energy proton scattering arises from the uncertainty in the NN range parameter $B_{A}$ for $p p$ and $p n$ amplitudes. The relevant relation between rms radii for the optical density and the proton and neutron matter distributions is $^{78}$ )

$$
r_{\text {opt }}^{2}=\frac{Z}{A}\left(r_{p}^{2}+6 B_{p p}\right)+\frac{N}{A}\left(r_{n}^{2}+6 B_{p n}\right) .
$$

Proton scattering data essentially fix the rms crtical radius $r_{\text {opt }}$ to about $\pm .02 \mathrm{fm}$ and electron scattering data $\mathrm{fix}$ the $\mathrm{rms}$ proton radius $\mathrm{r}_{\mathrm{p}}$. Thus, the imprecisely knowl quantities are the rms neutron radius $r_{n}$ and the isospin average NN range parameter,

$$
\bar{B}=\frac{Z}{A} B_{p p}+\frac{N}{A} B_{p n} .
$$

Since the relation is linear, uncertainties in $\bar{B}$ are directly translated into uncertainties in $r_{n}$, 


$$
\delta r_{n}=-\frac{6 \delta \vec{B}}{r_{n}}\left(\frac{A}{2 N}\right) .
$$

In fig. 1, the proton scattering data from ${ }^{40} \mathrm{Ca}$ is shown together with two equally good KMT optical potential fits labelled solution 1 and solution 2 based on NN range parameters differing by $\delta \bar{\beta}=0.1 \mathrm{fm}^{2}$. The differenco in rms neutron radii from solutions 1 and 2 may be estimated from eq. (19) to be $\delta r_{n}=0.17 \mathrm{fm}$, whereas the careful optical model calculations ${ }^{28}$ ) yield $\delta r_{n}=0.20 \mathrm{fm}$. Thus, the simple estimate is not misleading and one sees that the uncertainty $\delta \bar{\beta}$ must be considerably reduced before one can extract absolute neutron radif from proton diffraction data to much better than $\pm 0.1 \mathrm{fm}$ accuracy.

The situation is somewhat brighter for isotopic shifts because the NN range uncertainty cancels out to a degree. Figure 10 shows the ratio of ${ }^{48} \mathrm{Ca}$ and ${ }^{40} \mathrm{Ca}$ cross sections dramatizing the sensitivity to isotopic differences. Based on some simple assumptions, the analytic expressions of eqs. (13)(15) can be used to understand the basic features seen in fig. 10. For nuclear targets 1 and 2, the cross section ratio is approximately

$\frac{\sigma_{1}}{\sigma_{2}}=c e^{2 \pi q \Delta a}\left(1-\frac{\sin (q \Delta c) \sin (2 q \bar{c}+\bar{\phi})}{\sin ^{2}\left(q c_{2}+\frac{1}{2} \phi_{2}\right)+\sinh ^{2} \psi_{2}}\right)$

where $\Delta a=a_{1}-a_{2}, \Delta c=c_{1}-c_{2}, \bar{c}=\frac{1}{2}\left(c_{1}+c_{2}\right)$ and $\bar{\phi}=\frac{1}{2}\left(\phi_{1}+\phi_{2}\right)$ with $\phi$ and $\psi$ defined as in eqs. (14) and (15) for each nucleus. The cross section ratio involves tangent-like oscillations due to $\sin (2 q \bar{c}+\bar{\phi}) / \sin ^{2}\left(q c_{2}+1 / 2 \phi_{2}\right) \simeq 2 / \tan (q \bar{c}+1 / 2 \bar{\phi})$. The Coulomb interaction and the real to imaginary ratio $\rho$ of the $N N$ amplitude cause $\psi_{2}$ to be nonzero and this controls the amplitude of these oscillations. The tangentlike oscillations are centered on an exponentially increasing curve which is rather directly related to the diffusivity difference $\Delta a$ of the two-matter distributions. Detailed optical model fits to these data

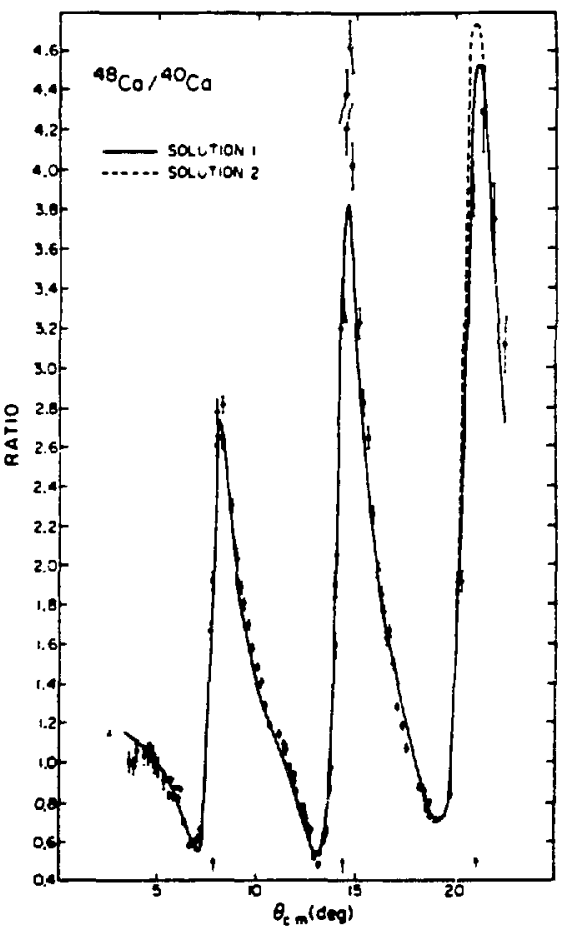
by L. Ray, et $a 1 .{ }^{28}$ ) are shown by the solid and dashed lines for two NN amplitude sets and the conclusion is that $r_{n}\left({ }^{48} \mathrm{Ca}\right)-r_{n}\left({ }^{40} \mathrm{Ca}\right)=0.16 \mathrm{fm} \pm .05 \mathrm{fm}$. Figure 11 shows neutron density differences deduced for $800 \mathrm{MeV}$ proton scattering from calcium isotopes compared with density matrix expansion (DME) theoretical predictions.$^{87}$ ) Again the chief contribution to the uncertainty is from the NN amplitudes. The solution $2 \mathrm{NN}$ amplitudes of Ray and the available phase shift analyses of 800 $\mathrm{MeV}$ NN scattering yield the range parameter $B_{\mathrm{A}} \approx .2 \mathrm{fm}^{2}$. However, this value leads to values of the neutron radius about $0.10 \mathrm{fm}$ smaller than expected from DME theories. The solution $1 \mathrm{NN}$ amplitudes of Ray employ $B_{A} \simeq .1 \mathrm{fm}^{2}$ and this value has been successful in fitting proton scattering data on a variety of nuclei with neutron radii closer to those expected from the DME theory.

Nucleon-nucleon amplitudes are generally considered to be better known in the $200 \mathrm{MeV}-500 \mathrm{MeV}$ energy range. The ambiguities encountered at $800 \mathrm{MeV}$ have motivated experiments in the $400-500 \mathrm{MeV}$ range where phase shifts are available and more conclusive tests of the multiple scattering theories are possible. 


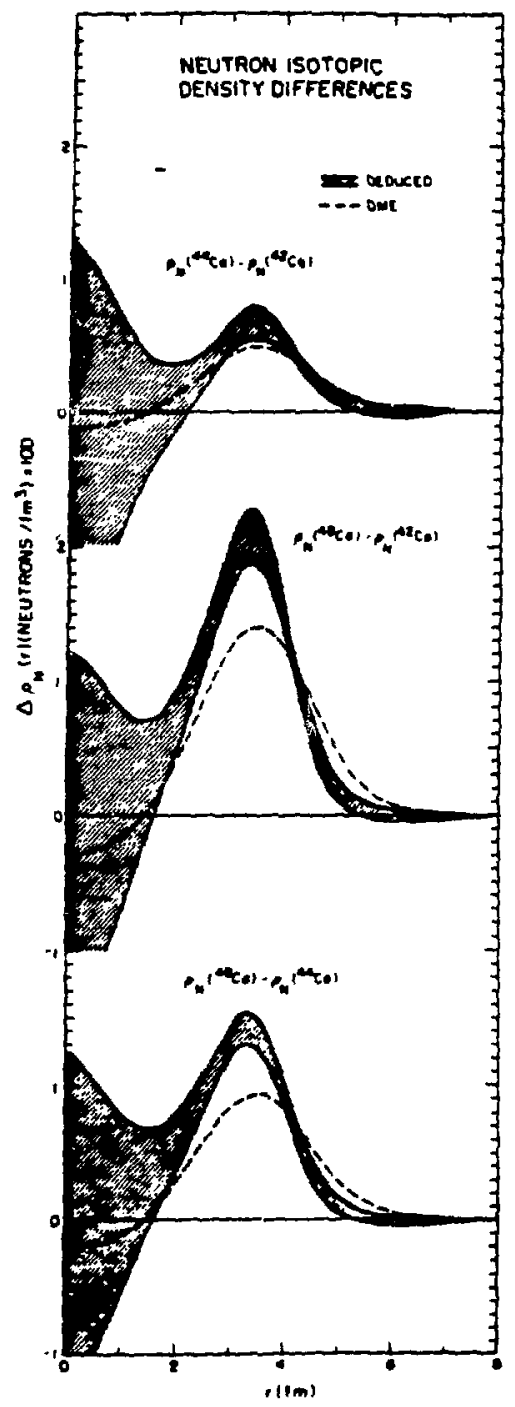

Fig. 11 Isotopic neutron density differences deduced by $800 \mathrm{MeV}$ proton scattering are shown by shaded areas. Density matrix expansion results (DME) are shown by dashed lines.

\section{Spin}

A general form for the spin 1/2 spin 0 scattering amplitude is $F(q)+\vec{\sigma} \cdot \hat{n} G(q)$, where

$$
\begin{aligned}
& F(q)=\frac{k}{i} \int_{0}^{\infty} d b \quad b \quad J_{0}(q b)\left[\frac{S_{F}^{(+)}(b)+S_{F}^{(-)}(b)}{2}\right] \\
& G(q)=k \int_{0}^{\infty} d b b J_{1}(q b)\left[\frac{S_{F}^{(+)}(b)-S_{F}^{(-)}(b)}{2}\right]
\end{aligned}
$$

The two Fourier-Bessel amplitudes $S_{F}^{( \pm)}$involve different geometries due to the spin dependence of $\mathrm{NN}$ amplitudes:

$$
f(q) /(2 i k)=A(q)+i \vec{\sigma} \cdot \vec{q} \times \hat{z} \quad C(q)
$$

For an uncorrelated nuclear density, the following simple form emerges for $z$ protons and $N$ neutrons ${ }^{73}$ ):

$$
S_{F}^{( \pm)}(b)=\left[i-\bar{A}_{p}(b) \pm \bar{C}_{p}^{\prime}(b)\right]^{2}\left[1-\bar{A}_{n}(b) \pm \bar{C}_{n}^{\prime}(b)\right]^{N}
$$


where $\bar{A}_{D}$ (b) and $\bar{A}_{n}(b)$ are defined as in eq. (12) for protons and neutrons. Spin functions $\bar{C}_{F}(b)$ and $\bar{C}_{n}(b)$ are defined in exactly the same fashlon excepl that the spin flip amplitude $C(q)$ from eq. (22) replaces the scalar amplitude $A(q)$. In eq. (23) the primes denote derivctives with respect to impact parameter.

"or large $q$ the scattering amplitudes may be approximated based on the asymptotic forms for the Bessel functions. The result is most simply expressed in terms of amplitudes $\mathrm{F}^{( \pm)}$for spin-up and spin-down scattering:

$F^{( \pm)} \equiv F \pm G \because \frac{k}{i}\left(\frac{2}{\pi q}\right)^{1 / 2} \int_{0}^{\infty} d b b^{1 / 2}\left(e^{ \pm i(q b-\pi / 4)} S_{F}^{(+)}(b)+e^{\mp i(q b-\pi / 4)} S_{F}^{(-)}(b)\right)$.

In addition to the usual polarization parameter $P$, Glauber and 0 sland ${ }^{4}$ ) have pointed out a second parameter $Q$ related to spin rotation. These two parameters are the real and imaginary parts of the scalar-spin flip interferences:

$$
P+i Q=\frac{\left|F^{(+)}\right|^{2}-\left|F^{(-)}\right|^{2}+2 i\left|F^{(+)}\right|\left|F^{(-)}\right| \sin \phi_{+}}{\left|F^{(+)}\right|^{2}+\left|F^{(-)}\right|^{2}}
$$

where $\phi_{+}$- is the relative phase of $F^{(+)}$and $F^{(-)}$. Thus $P$ determines the cross section difference for spin-up and spin-down scattering and $Q$ determines the relative phase of spin-up and spin-down amplitudes.

In a simple but instructive limit where only the first-order spin-flip correction is retained and the flip amplitude $C(q)$ is proportional to the scalar amplitude $A(q)$, the observables $P$ and $Q$ are sinooth functions of $q$ determined entirely by the forward $N N$ amplitudes $A_{0}$ and $C_{0}$ in almost the same fashion as for NN scattering 50,51 ):

$$
P+i Q=\frac{2 q A_{0}\left[i C_{0}\right]^{*}}{\left|A_{0}\right|^{2}+q^{2}\left|C_{0}\right|^{2}}
$$

The polarization data summarized in fig. 2 have tangent-1ike oscillations superimposed on a smooth background essentially determined by eq. (26). The explanation of the oscillacions has been given by Amado, McNeil and Sparrow ${ }^{88}$ ) based on applying the saddle point method to the spin amplitudes to develop analytic formulas. When the spin terms $\bar{C}^{\prime}(b)$ in eq. (23) can be treated as a perturbation of the dominant scalar terms involving $\bar{A}(b)$, Amado, McNeil and Sparrow argue that the saddle points determined in eq. (11) are simply shiftet. For the part of eq. (24) involving $S_{F}{ }^{(+)}(b) \approx \exp \left(-\bar{A}(b)+\bar{C}^{\prime}(b)\right)$, where $\bar{A}$ and $\bar{C}$ are isospinaveraged quantities, there are two saddle points given by

$$
b_{ \pm}^{(+)}=b_{ \pm}+w\left(1+\frac{\delta_{ \pm}}{c \pm i \pi a}\left(1-3\left[\frac{g(c \pm i \pi a)}{-a}\right]^{2 / 3}\right)\right)
$$

Similarly for the part of pq. (24) involving $S_{F}^{(-)}(b) \simeq \exp \left(-\bar{A}(b)-\bar{C}^{\prime}(b)\right)$ there are two saddle points, $b-{ }^{-}$, given also be eq. (27) but with the sign of $w$ reversed. The complex length parameter $\delta_{ \pm}$represents the geometry difference between $\bar{A}(b)$ and $\bar{C}(b)$ functions. Using eq. (16), this geometry difference may be directly re? ated to the NN amplitude range parameters $B_{A}$ and $B_{C}$ as follows:

$$
\delta_{ \pm}=\left(\beta_{c}-\beta_{A}\right)\left(\frac{2}{c+\frac{7}{15}\left(\pi^{2} a^{2} / c\right)} \pm \frac{3 i}{\pi a}\right) \text {. }
$$

Analytic results for the spin-up and sp:Ln-down scattering amplitudes of eq. (24) follow in a straight forward manner.

The dashed line in $\mathrm{fig}$. 12 shows a calculation of the $\mathrm{p}-208 \mathrm{~Pb}$ polarization bas $d$ on these results, but omitting the impertant Coulomb effects. Two complex length parameters govern the polarization. The first is $w=C_{0} / A_{0}$ which has been taken to be $w=-0.16-i 0.20 \mathrm{fm}$ based on suitably averaging the pp and pn amplitudes obtained from Arndt's phase shifts ${ }^{89}$ ). The second complex length, 
Fig. 12 Asymptotic approximation to polarization for elastic scattering of $800 \mathrm{MeV}$ protons by $20{ }^{\mathrm{Pb}}$. Experimental data äre shown by dots.. Soild line shows prediction of data-to-data relation,

$\delta_{ \pm}=0.08 \pm 10.15 \mathrm{fm}$, has been $f i t$ to the polarization data. The parameter $w$ governs the rise to the polarization that is similar to NN scatteing, eq. (26), while the geometry difference parameter $\delta_{ \pm}$is responsible for the tangentlike oscillations. The

lengths $\delta \pm$ should also be related to the $\mathrm{NN}$ amplitude parameters as in eq. (28) keeping in mind that the range parameters $B_{C}$ and $B_{A}$ are generally complex. This alters the analysis of reference because $\delta_{+} \neq \delta_{-}{ }^{*}$. A recent phase shift analysis at $0.8 \mathrm{GeV}$ leads to a prediction $\delta_{+}=-.173-i .001 \mathrm{fm}$ and $\delta_{-}=.161+i .065 \mathrm{fm}$, both of which are far from the effective values obtained by fitting the $208 \mathrm{pb}$ data. Amado, McNeil and Sparrow ${ }^{88}$ ) develop simple explanations of the characteristic tangent-like oscillations and they also point out a contribution proportional to $1 / \sigma$, where $\sigma$ is the elastic cross section, that seems to be new to polarization phenomenology. They show that the geometry difference between scalar $\bar{A}(b)$ and spin $\overline{f l i p} \bar{C}(b)$ functions is crucial to understanding the spin measurements. The polarization is also expressed in terms of the elastic scattering cross section in a "data-to-data" relation. Altnough this approach loses contact with the underlying dynamics, it does provide a much betcer fit to the polarization as indicated by the solid line in fig. 12.

Using nonasymptotic methods, Fäldt and Ingemarsson 52) have also developed approximate expressions for the polarization observables reaching some of the same conclusions. Faldt and Ingemarsson ${ }^{53}$ ) have incorporated both Coulomb and magnetic effects in their analysis of $\mathrm{p}-208 \mathrm{~Pb}$ polarization as indicated in fig. 13. The Coulomb effects fill the dramatic minima and the magnetic effects are also important as was pointed out earlier by Osland and Glauber ${ }^{46}$ ). A recent KMT optical potential calculation by $L$. Ray also indicates the importance of magnetic spin flip effects. By comparison of figs. 12 and 13, one sees that the "data-todata" relation in fig. 12 has mainly restored the missing Coulomb effects.

The above review has focused on the simple insights to high energy proton scattering which follow from the development of direct relations between the optical densities and the observed cross sections and polarizations. Additional insights should be mentioned as well--such as the development of simple relations between elastic and inelastic cross sections based on approximate models for the inelastic transition density $90-91$ ). Since the scattering is dominated by the "bright spots", many of the features of inelastic scattering can be quite simply explained using the Tassie model ${ }^{92}$ ) for collective transition density in the nuclear surface. The dynamical issues in proton scattering are considerably clarifled by the asymptotic methods. Proton scattering measurements have an interferometric sensitivity :o certain combinations of nuclear length parameters and NN amplitude parameters. Although firm conclusions cannot be drawn at present, there is clearly a trend towards finding that the free NN amplitudes and standard nuclear geometry parameters do not yield good fits to the data, but that effective NN amplitude parameters can be found that do fit the data. Preliminary 
analyses of the first measurements of the spin rotation parameter $Q$ at $0.5 \mathrm{GeV}$ also support this trend.

Fig. 13 Polarization ( $P$ ) and spin rotation ( $Q$ ) for elastic scattering of $800 \mathrm{MeV}$ protons by ${ }^{208} \mathrm{pb}$. Dashed line omits Coulomb effects and dotted line omits magnetic effects.

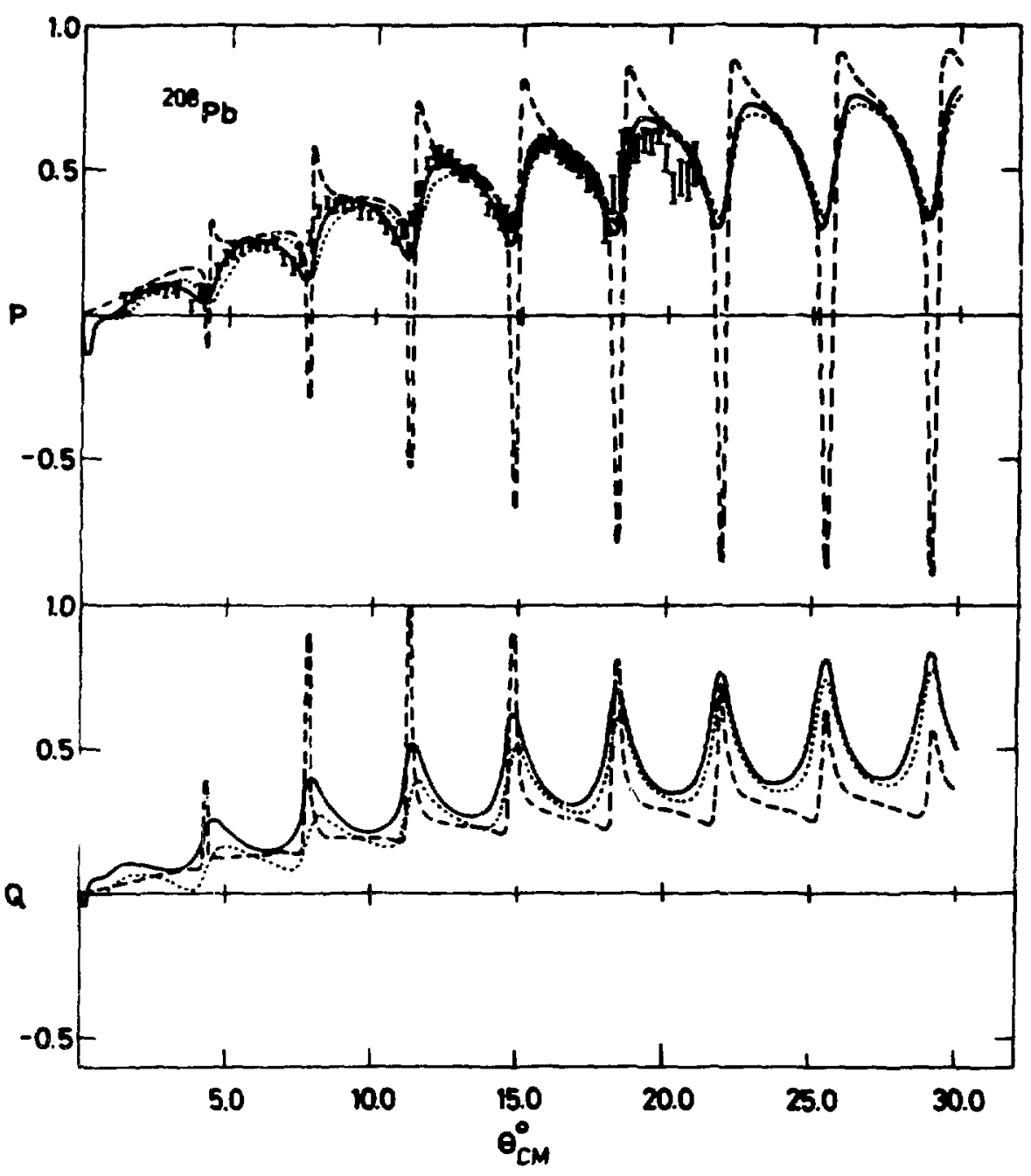

4. NN amplitudes

The most obvious uncertainties in theoretical analyses of proton scattering continue to be the NN amplitudes, however, recent progress in NN scattering has been impressive. Much of the interest has been driven by the possibility that dibaryons might be the explanation of the quite unexpected and large spin dependence discovered in Argonne National Laboratory measurements of $\Delta \sigma_{\mathrm{L}}$ and $\Delta \sigma_{\mathrm{T}}$. $A$ very nice review of the spin-dependent cross section measurements has recently been given by $H$. Spinka ${ }^{93}$ ). However, the opening of the $\Delta$-isobar production channel in NN scattering provides a simpler explanation of the basic features of the data94-96). Phase shift analyses for energies above $300 \mathrm{MeV}$ are now available from three sources ${ }^{9-99}$ ) although the data base, particularly for np scattering, is not yet very good. In multiple scattering calculations simple assumptions about NN amplitudes are often employed or, alternatively, "effective" NN amplitude parameiers are self-consistently deduced by optimizing fits to selected protonnucleus data. In this section, some information regarding the "free" and "effective" NN amplitudes is reviewed. A more extensive $r$ criew with tables of NN amplitudes is contained in ref. 78. A recent paper by Love and Franey100) provides simple forms for the NN $t$ matrix to $800 \mathrm{MeV}$ for use in distorted wave calculations.

A parameterization of the $\mathrm{NN}$ amplitude that is convenient for multiple scattering calculations is 


$$
\begin{aligned}
(21 k)^{-1} f(q)=A(q) & +1\left(\vec{\sigma}_{1}+\vec{a}_{2}\right) \cdot \vec{q} \times \dot{z} C(q)+\vec{\sigma}_{1} \cdot \vec{\sigma}_{2} B(q) \\
& +\vec{\sigma}_{1} \cdot \vec{q}_{\sigma_{2}} \cdot \vec{q} D(q)+\sigma_{12} \sigma_{2 z} E(q)
\end{aligned}
$$

where $A(q)=A_{0} e^{-B_{A} q^{2}}, C(q)=C_{0} e^{-B_{c} q^{2}}$, etc., and $\vec{q}$ is the momentum transfer and $\hat{z}$ is the average momentum direction. The double spin flip amplitudes $B, D$ and $E$ do not play a significant role in elastic scattering by spin-zero nuclei, however they can be quite important in selected inelastic transitions. At forward angles, convenient forms for these amplitudes omitting Coulomb and magnetic spin flip effects are ${ }^{78}$ ):

$$
\begin{aligned}
& A_{0}=(8 \pi)^{-1}=(1-i .) \\
& C_{0}=w A_{0} \\
& B_{0}=-(16 \pi)^{-1} \therefore \sigma_{T}\left(1-i_{T}\right) \\
& E_{0}=-(16 \pi)^{-1}\left[\therefore \sigma_{L}\left(1-i_{L}\right)-2 \sigma_{T}\left(1-i_{T}\right)\right]
\end{aligned}
$$

and

$$
D_{0}=-\frac{1 g^{2} /(4 \pi)}{4 k_{2} \sqrt{s_{2}} m^{2}} .
$$

The double spin flip amplitudes $B_{0}$ and $E_{0}$ are directly related to the cross section differences $\Delta O T$ and $\Delta c_{L}$ in transverse and longitudinal spin states. Dispersion calculations in pure spin channels ${ }^{10 l}$ ) have been used to calculate the associated real to imaginary ratios $\rho T$ and $\rho_{L}$ which tend to be large (typically 3 to 4 ). The $D$ amplitude is dominated by one pion exchange at small $q$.

Due to the sizeable double spin flip terms, it is not possible to extract the more important scalar (A) and spin flip (C) amplitudes without a complete set of measurements.

Figure 14 shows a typical example where phase shift analysis ${ }^{99}$ ) has been used to determine the five complex amplitudes. The differential cross section is built up by the incoherent sum,

$$
\frac{d \sigma}{d t}=4 \pi\left(|A|^{2}+2 q^{2}|C|^{2}+|B|^{2}+\left|B+q^{2} D\right|^{2}+|B+E|^{2}\right),
$$

and the polarization is given by

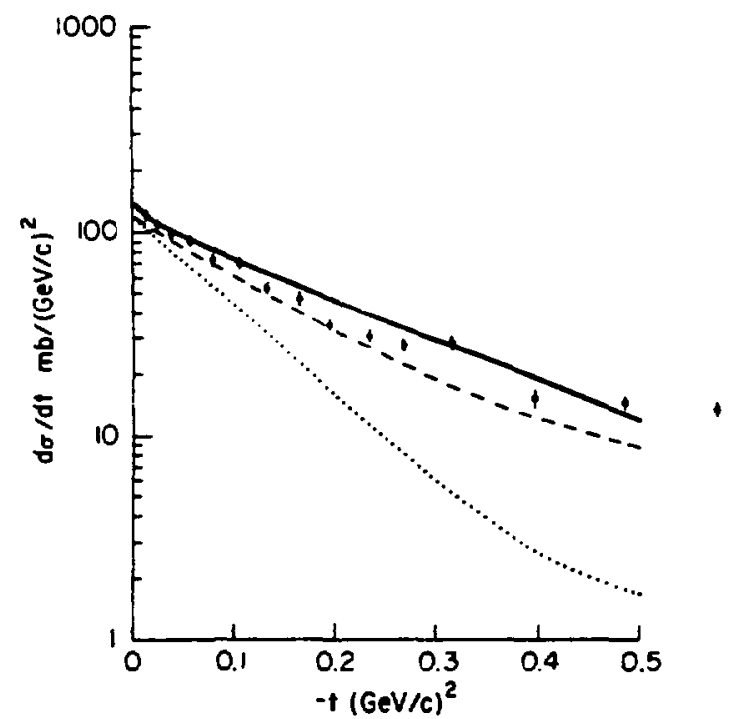

$$
P=8 \pi \operatorname{Re}\left[(A+B)(i q C)^{*}\right] /(d \sigma / d t) .
$$

The slope parameter of the differential cross section fit according to $\exp \left(-2 B_{C} q^{2}\right)$ is $B_{0}=.1 \mathrm{fm}^{2}$ while the slope parameter of just the scalar amplitude is about twice as big: $s_{A}=.2 \mathrm{fm}^{2}$. The absence of phase shift amplitudes has of ten left no alternative to the simple assumption $B_{A}=B_{\sigma}$ in many proton scattering analyses,

Fig. 14 Proton-proton differential cross section at $1 \mathrm{GeV}$ based on eq. (31) for Hoshizaki phase shifts - Dashed line omits double spin flip amplitudes $B, D, E$. Dotted line omits all spin amplitudes. 
however, it now becomes clear that substantial differences must be expected. As discussed above, the difference between $B_{\sigma}$ and $B_{A}$ is very relevant to neutron radius determination. Figure 15-illustrates that the double spin flip B amplitude in eq. (32) plays a significant role in determining the polarization. One of the implications is that the spin flip range parameter $f_{c}$ can only be reliably extracted when all the NN amplitudes are known.

Three recent phase shift analyses have been used in ref. 78 tc extract the Gaussian NN parameters. For the scalar and spin flip amplitudes, the Gaussian approximation is generally accurate for $q \leqslant 2 \mathrm{fm}^{-1}$ provided the slope parameters are complex. Table I lists some NN amplitude parameters at $500 \mathrm{MeV}$. The Bugg, et al. phase shifts ${ }^{97}$ ) are based on extensive measurements at TRIUMF at $515 \mathrm{MeV}$. The Hoshizaki ${ }^{99}$ ) and the Arndt ${ }^{98}$ ) phase shift and solutions used in this comparison did not include all the data. Thus, the table represents the staius of $\mathrm{NN}$ amplituder near $500 \mathrm{MeV}$ as of about the beginning of 1980 and reasonable but far from precise agreement of the three phase shifts is apparent. One surprise that emerges from the phase shift amplitudes is the need for large imaginary parts to the slope parameters $B_{A}$ and $B_{C}$. As may be seen from eq. (28) this implies substantial geometric differences between the scalar and spin flip optical densities at $500 \mathrm{MeV}$.

\begin{tabular}{|c|c|c|c|c|}
\hline & $\begin{array}{l}\text { e I: Compari } \\
{ }_{A_{0}}(\mathrm{GeV} / \mathrm{c})^{-2}\end{array}$ & $\begin{array}{l}\text { of } 500 \mathrm{MeV} \mathrm{ph} \\
e_{\mathrm{A}}(\mathrm{GeV} / \mathrm{c})^{-2}\end{array}$ & $\begin{array}{l}\text { shift amplitu } \\
C_{0}(\mathrm{GeV} / \mathrm{c})^{-3}\end{array}$ & ${ }_{\mathrm{C}}(\mathrm{GeV} / \mathrm{c})^{-2}$ \\
\hline $\begin{array}{l}\text { Bugg, et a1.97) } \\
(.515 \mathrm{GeV})\end{array}$ & $3.24-10.74$ & $4.27-i 3.93$ & $-1.43-i 6.04$ & $1.66+i 0.0$ \\
\hline $\begin{array}{l}\left.\text { Arndt }{ }^{98}\right) \\
\quad(.508 \mathrm{GeV})\end{array}$ & $3.22-i 1.41$ & $5.35-i 6.78$ & $-1.39-i 6.70$ & $2.55+i 0.33$ \\
\hline $\begin{array}{c}\text { Hoshizaki } \\
(.508 \mathrm{GeV})\end{array}$ & $3.08-i 1.31$ & $4.39-i 5.20$ & $-1.76-i 6.55$ & $3.01+i 0.68$ \\
\hline
\end{tabular}

An important point regarding the role of $\mathrm{NN}$ amplitudes in proton scattering is that they generally enter as in eq. (12) multiplied by a nuclear form factor. Because the form factor decreases rapidly with $q$, the NN amplitudes are primarily influential at small q $\leqslant 2 \mathrm{fm}^{-1}$. This means that accurate NN amplitudes at forward angles $\theta_{\mathrm{cm}} \leqslant 40^{\circ}$ are needed as input to proton-nucleus analyses, although for light nuclei the $\mathrm{NN}$ amplitudes at larger $q$ also are important. One of the best ways to ootain PP amplitudes at small angles is to use a proton spectrometer as has been done in a recent Los Alamos experiment.102)

Table II lists some representative pp amplitude parameters which have been used in analyses of $1 \mathrm{GeV}$ proton-nucleus data including polarizations. Chaumeaux, Layly and Schaeffer (CLS) ${ }^{63}$ ) and also Alkhazov, Belostotsky and Vorobyov (ABV) ${ }^{73}$ ) have obtained fits for heavy nuclei using relatively simple assumptions about NN parameters. An analysis of $1 \mathrm{GeV} \mathrm{p}^{-4} \mathrm{He}$ scattering by Wallace and Alexander (AWH) ${ }^{103}$ ) was based on pp amplitude from Hoshizaki's phase shift analysis, 
however, the pn spin flip amplitudes were f1t to the ${ }^{4}$ He data. Finally, the 11 st Includes amplitude parameters deduced by McNeil ${ }^{104}$ ) in an analysis of $1 \mathrm{GeV} p-d$ scattering. Thus, each set of amplitudes in the table provides a good fit to one or more nuclei.

\begin{tabular}{|c|c|c|c|c|}
\hline Táble II: Com & $\begin{array}{l}\text { son of } 1 \mathrm{GeV} \\
\mathrm{A}_{0}(\mathrm{GeV} / \mathrm{C})^{-?}\end{array}$ & $\begin{array}{l}\text { Plitudes used } \\
E_{A}(\mathrm{GeV} / \mathrm{C})^{-2}\end{array}$ & $\begin{array}{l}\text { in proton-nucl } \\
\mathrm{C}_{\mathrm{C}}(\mathrm{GeV} / \mathrm{c})^{-3}\end{array}$ & $\begin{array}{l}\text { analyses } \\
B_{c}(\mathrm{GeV} / \mathrm{c})^{-2}\end{array}$ \\
\hline $\begin{array}{l}\text { Chaumeaux-Layly- } \\
\text { Schaeffer (CLS) }\end{array}$ & $4.88+10.0$ & $2.50+i 0.0$ & $0.0-13.78$ & $4.45+10.0$ \\
\hline $\begin{array}{l}\text { Alkhazov-Belostotsky- } \\
\text { Vorobyov (ABV) }\end{array}$ & $4.84+i 0.24$ & $2.76+i 0.0$ & $1.60-i 3.20$ & $8.6+i 0.0$ \\
\hline $\begin{array}{l}\text { Alexander-Wallace- } \\
\text { Hoshizaki (AWH) }\end{array}$ & $4.79+10.88$ & $4.39-i 0.24$ & $-3.02-15.01$ & $3.43+i 0.0$ \\
\hline McNeil (M) & $4.88-i 0.5$ & $2.65+i 0.0$ & $2.47-14.97$ & $3.8+i 0.0$ \\
\hline
\end{tabular}

Figures 16 and 17 compare the predictions for $1 \mathrm{GeV}$ p-d scattering based on each set of NN parameters in this table 104 ).
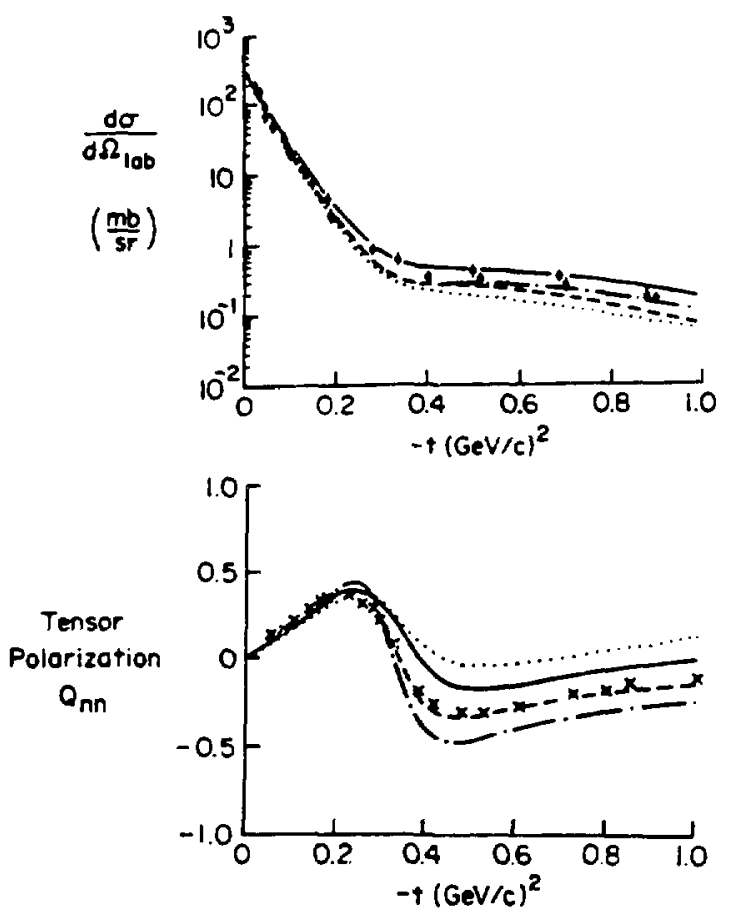

Fig. 16 Differential cross section and tensor polarization $Q_{\mathrm{nn}}$ data for $1 \mathrm{GeV}$ p-d elastic scatteringl05) are compared with predictions based on $\mathrm{NN}$ amplitudes of Table II:104)
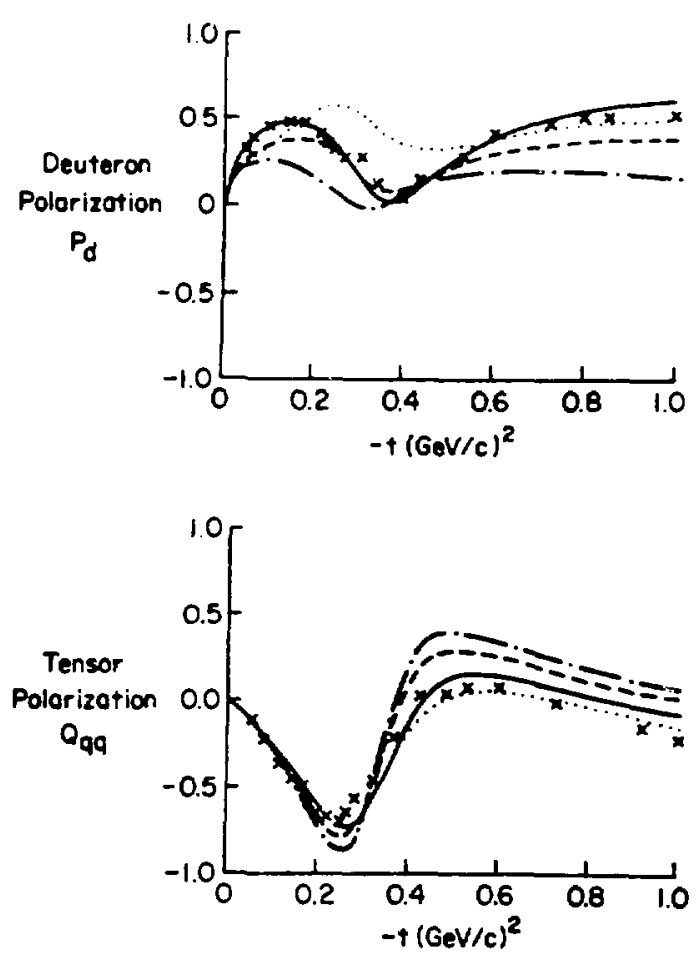

Fig. 17 Tensor polarization data for $1 \mathrm{GeV}$ p-d elastic scattering. Lines have some weaning as in fig. 16 . 


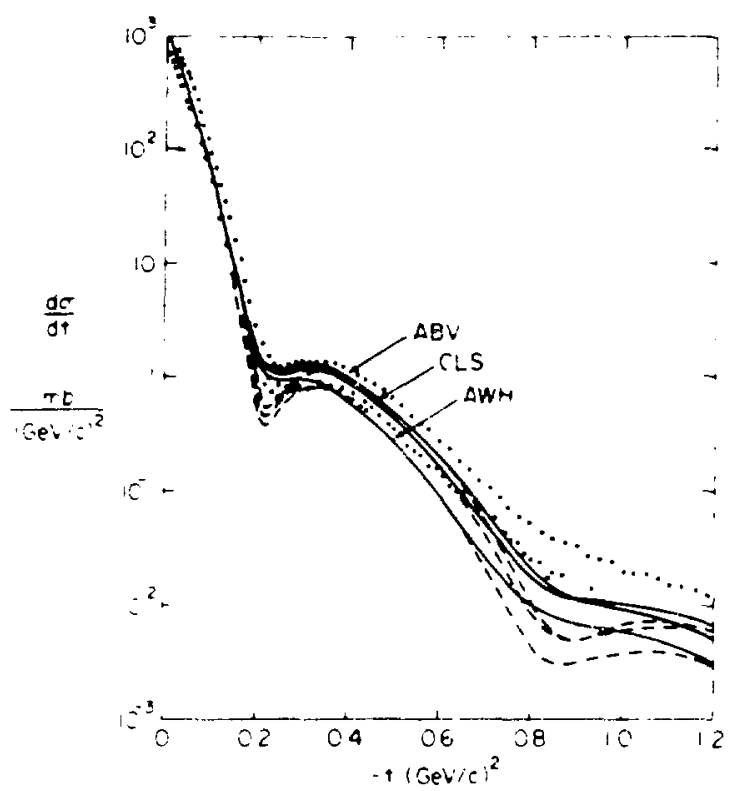

Fig. 18 Differential cross section data (upper points refs. 15, 7; lower points refs. 107, 108.) with predictions based on Gaussian NN amplitude parameters of Table II. Dashed line shows Glauber calculation while solid lines include intermediate isobar effects.

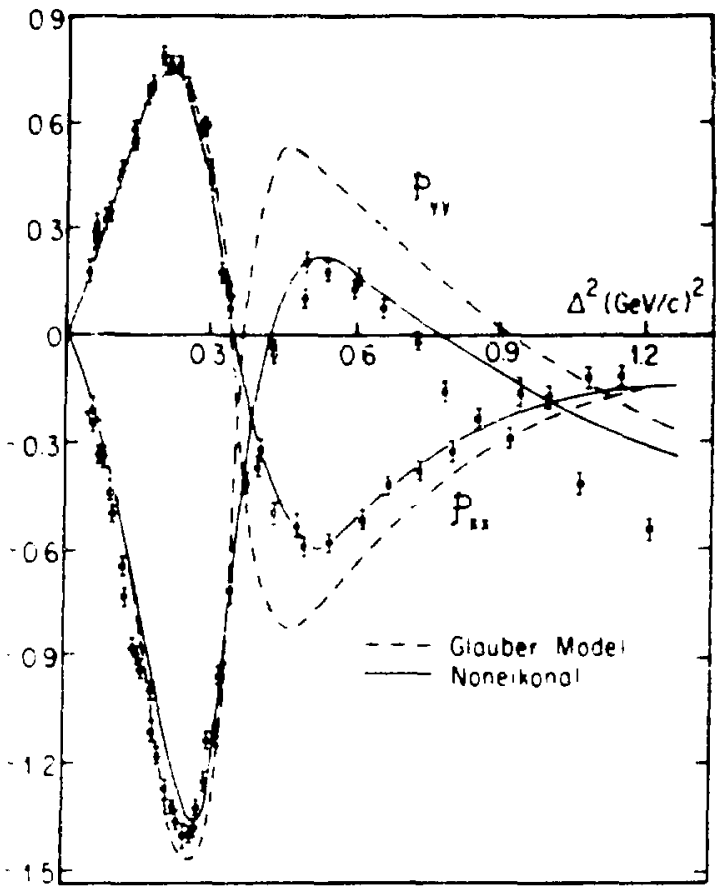

Fig. $191 \mathrm{GeV}$ p-d elastic scattering tensor polarization predictions with and without noneikonal effects ${ }^{105}$ ).

Figure 18 shows simllar predictions for $1 \mathrm{GeV} \mathrm{p}-{ }^{4} \mathrm{He}$ scattering ${ }^{78}$ ). One point of these comparisons is that no one set of NN parameters has yet been successful in fitting both light and heavy nucleus scattering data. The second point is that light ion scattering is very sensitive to the NN amplitude parameters and therefore provides a very important testing ground for the protonnucleus dynamics. An analysis by Alberi, et al.105), fig. 19, has recently pointed out that noneikonal effects are important in calculations of $p-d$ spindependent observables.

Multiple scattering theory predictions are generally in qualitative agreement with proton-nucleus data, however, the differences in details can be large. It remains unsettled whether the "free" NN amplitudes become significantly modified in the nucleus, however the non-negligible spin effects suggest that the interaction should be modifled by Pauli effects. In light ion scattering, there is a significant modification of a kinematical nature due to the NN amplitude being in the Breit frame rather than the NN center-of-mass frame ${ }^{117,78}$ ). Also, there is a significant contribution to $1 \mathrm{GeV}$ p- ${ }^{4} \mathrm{He}$ scattering due to isobar intermediate states as is evidenced by the difference of solid and dashed curves in fig. 18.

\section{5. $\Delta$-isobars}

The proton-nucleus reaction mechanism at intermediate energies is generally assumed to be dominated by a sequence of quasi-free NN scatterings. Above the inelastic threshold, this means that there is a substantial $\Delta$-production probability with subsequent decay of the $\Delta$ to $\pi N$ states. Recent isospin analyses by VerWest and Arnd ${ }^{109}$ ) suggest that all of the free $N N \rightarrow N N \pi$ reactions can be understood in terms of an intermediate $\mathrm{N} \Delta$ state in the energy region 0.5 to $1.0 \mathrm{GeV}$. 

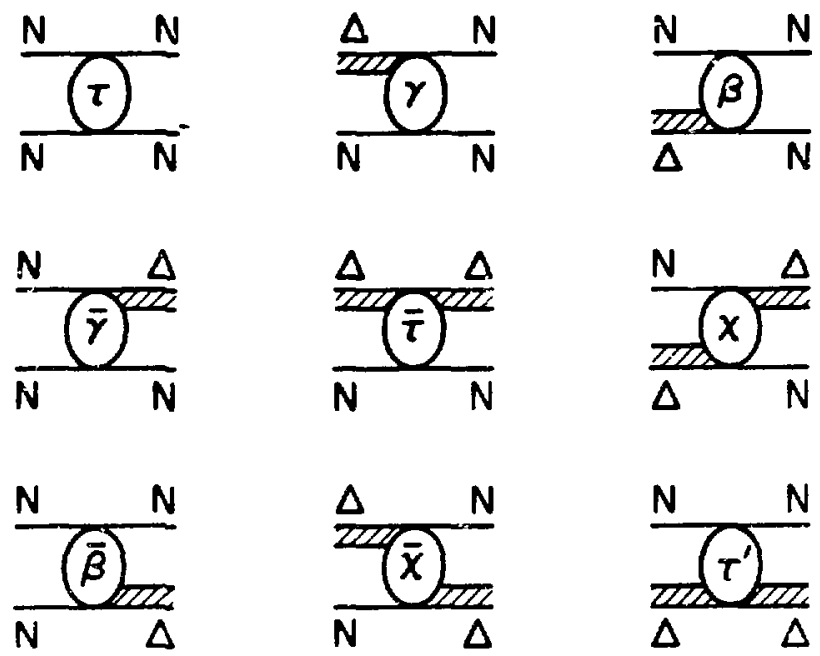

Fig. 20 Coupled-channel t-matrtix elements for $N N, \Delta N$ and $N \Delta$ channels.

A dynamical model of NN scattering based on coupled $N N, N \Delta$ and $\Delta \mathrm{N}$ channels is illustrated in fig. 20. In this model the nucleon-nucleon $t$ matrix is represented by a $3 \times 3$ matrix in which the usual $\mathrm{NN} t$ matrix appears as the upper left entry. A static model of the isobar is assu..ed 110,111 ) and the threedimensional dynamical equations are chosen to incorporate approximate cancellations between crossed and uncrossed Feynman diagrams. A detailed

discussion of this model is given in ref. 78 and therefore the present review will focus on qualitative aspects of the theory. Related work on a Hamiltonian theory of $\pi-N-\Delta$ systems is also given in a recent paper by Betz and Lee ${ }^{112}$ ).

The distinction between $\Delta \mathrm{N}$ and $\mathrm{N} \Delta$ channels is important in proton-nucleus collisions since in the $\Delta \mathrm{N}$ case, the $\Delta$-isobar carries the projectile proton's momentum and in the $\mathrm{N} \Delta$ case, a nuclear $\Delta$-excitation is made. The nuclear $\Delta$-excitations are, of course, closely related to the isobar-hole states produced in pion scattering.

A multiple scattering theory based on the coupled-channel NN dynamics follows in a straightforward fashion in terms of a Watson-type expansion for the $3 \times 3 \hat{T}$ matrix:

$$
\hat{I}=\sum_{i} \hat{\tau}_{i}+\sum_{i} \sum_{j \neq i} \hat{\tau}_{i} \hat{G} \hat{\tau}_{j}+\ldots
$$

where each two-body operator, $\hat{\tau}_{i}$, is a $3 \times 3$ matrix as in $\mathrm{f} i g .20$. The $3 \times 3$ matrix $\hat{G}$ is diagonal with elements describing the propagation of a nucleon or a $\Delta$, depending on which channel carries the projectile proton's momentum, in the presence of a nuclear or isobaric excitation of the residual system.

In this formalism, the usual $T$ matrix for proton-nucleus scattering must be projected from $\hat{\mathrm{T}}$ by demanding that the initial and final states have no isobars present and this causes the single scattering terms ol eq. (33) to involve just the NN $t$ matrix as in the usual Watson multiple scattering expansion. The double scattering terms contain new contributions due to three-body isobar intermediate state effects that cannot be included in the free NN $t$ matrix. Originally discussed in relation to inelastic shadowing corrections for total proton-nucleus cross sections at high energy 13-115), these intermediate isobar effects were observed by Ikeda ${ }^{16}$ ) to yield a significant correction to $\mathrm{p}^{-4} \mathrm{He}$ angular distributions in the $1 \mathrm{GeV}$ energy region. Figure $21 \mathrm{a}$ illustrates one intermediate isobar contribution. Although the formalism suggests that fig. 2la be viewed as a $\Delta-$ excitation created on nucleon $j$ and destroyed on nuclion $i$, an alternative and completely equivalent view is that of $\mathrm{fig} .2 \mathrm{lb}$ in which the incident proton is viewed as scattering in resonant fashion from a $T=1$ meson current being exchanged by nucleons $i$ and $j$. Thus, the intermediate $\Delta$-states are related to meson exchange currents. A second type of intermediate isobar state is indicated in $\mathrm{fig}$. $21 \mathrm{c}$, or equivalently in $\mathrm{fig}$. $21 \mathrm{~d}$, in which a nuclear $\Delta$-excitation is created and later destroyed. Because a spin-isospin correlatic is needed for either process $a$ or $c$ to contribute to proton elastic scattering, the two tend to be equivalent even though process $c$ involves nucleon exchange in the intermediate state. 


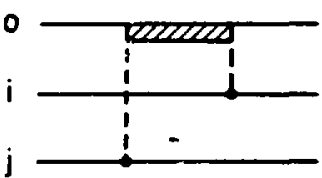

(a) $\gamma_{i} \bar{G} \bar{\gamma}_{j}$

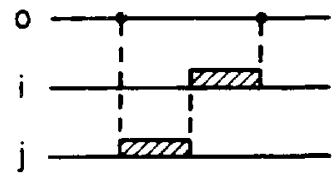

(c) $\beta_{i} G^{\prime} \bar{\beta}_{j}$

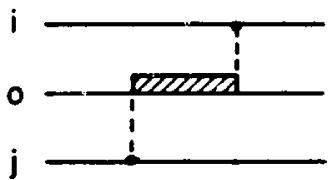

(b) $\gamma_{i} \bar{G} \bar{\gamma}_{j}$

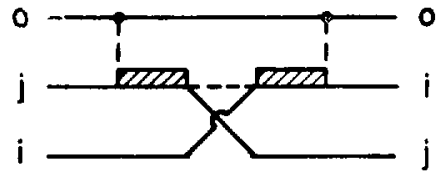

(d) $\beta_{i} G^{\prime} \bar{\beta}_{j}$

Fig. 21 Intermediate isobar states involving the projectile $(0)$ and two target nucleons ( 1 and $j$ ). See text for discussion.

Although the NN amplitudes are not well known at $1 \mathrm{GeV}$ and even less is known about the $\mathrm{NN} \rightarrow \mathrm{N} \Delta$ and $\mathrm{N} \Delta \rightarrow \mathrm{N} \Delta$ amplitudes involved in the coupled-channel multiple scattering theory, calculations based on reasonable assumptions have indicated a significant intermediate isobar contritution to $1 \mathrm{GeV}$ $\mathrm{p}-{ }^{4} \mathrm{He}$ scattering. Figure

22 shows the result of calculations by Wallace and Alexander ${ }^{103}$ ) who find that the effect of intermediate isobar states is to fill the interference minimum which generally shows up in multiple diffraction calculations without intermediate isobar effects. The possibility that multiple scattering of the $\Delta-$ isobar could reduce the intermediate isobar contribution of fig. 2la has been pointed out by Auger, Lazard and Lombard 118) in a contribution to this conference. Because of amplitude uncertainties, particularly with respect to the phases of the $\mathrm{NN} \rightarrow \mathrm{N} \Delta$ amplitude and the magnitude and phase of the $\mathrm{N} \Delta \rightarrow \mathrm{N} \Delta$ amplitude, the evidence for intermediate isobar contributions to $\mathrm{p}-{ }^{4} \mathrm{He}$ remains inconclusive but interesting. Although the intermediate isobar effect is thought to be small in heavy nuclei for $\mathrm{N}=\mathrm{Z}$, it does provide a specific correction in the $T=1$ NN channel which has yet to be considered in analyses of isotopic shifts of neutron radil. Another, perhaps more important, type of intermed late isobar effect arises due to the twobody $N \Delta$ intermediate states in the NN $t$ matrix.

Because the intermediate isobar states are reached by a $T=1, J=1$ exchange, the Pauli principle must modify the intermediate $\Delta \mathrm{N}$ of the $\mathrm{NN} t$ matrix in the nuclear medium. Also, there are well-known spreading effects for the nuclearisobar states 119,120 ) which are excited as intermediate states

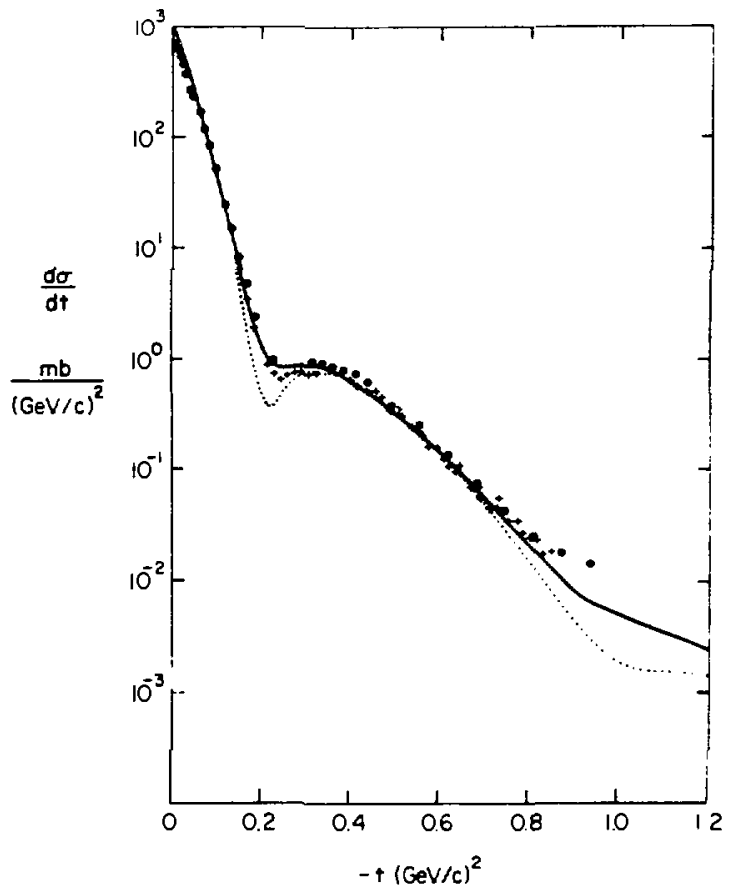

Fig. 22 Effect of intermediate states in $1 \mathrm{GeV} \mathrm{p}-{ }^{4} \mathrm{He}$ differential cross section. Dotted line shows Glauber calculation omitting isobar intermediate states. Kinematic transformation of the NN amplitudes 117,78 ) fills the second minimum near $-t=0.9$ which is present in fig. 18 . of the $\mathrm{NN} t$ matrix. Other isobar related effects of potential importance arise from higher-order terms in the multiple scattering expansion. Figure 23 illustrates some third-order terms, 


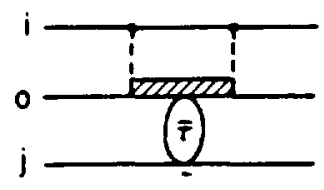

(a) $\gamma_{i} G \overline{\tau_{j}} G \bar{y}_{i}$

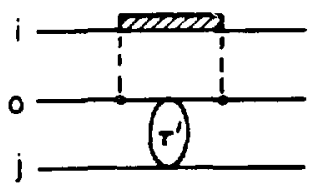

(b) $\beta_{i} G^{\prime} \tau_{j}^{\prime} G^{\prime} \bar{\beta}_{i}$

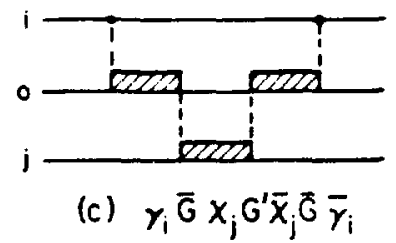

Fig. 23 Local field corrections due to interactions which modify the Intermedlate states of the NN $t$ matrix due to the presence of a third nucleon.

called local fleld corrections, which act to modify the $\mathrm{NN} t$ matrix in the nuclear medium. In fig. 23a the intermediate isobar state in the scattering of the projectile (labelled 0 ) and nucleon $i$ is modifed by scattering of the isobar by a third nucleon (labelled $\mathrm{j}$ ). Figure $23 \mathrm{~b}$ is a similar process except that the intermediate state nucleon scatters from a third nucleon.

Finally, fig. 23c indicates a more complicated local field correction due to $\Delta$-exchange in the intermediate states. All of these nuclear medium corrections to the NN $t$ matrix can be organized into an effective $N N$ matrix in the nucleus in principle $\mathrm{e}^{12 l}$ ), however a prerequisite to meaningful calculations of the effective $t$ matrix is a satisfactory model of the free $N N$ matrix based on the coupledchannels approach.

Direct signals for the importance of isobars in proton-nucleus scattering are obtained in inclusive $\left(p, p^{\prime}\right)$ experiments ${ }^{122}, 123$ ) which extend to large momentum loss. Figure 24 shows recent $800 \mathrm{MeV}$ data for protons inelastically scattered by ${ }^{2} \mathrm{H}$ and ${ }^{12} \mathrm{C}$. The $\Delta$-production shows up as a broad peak at large energy loss in
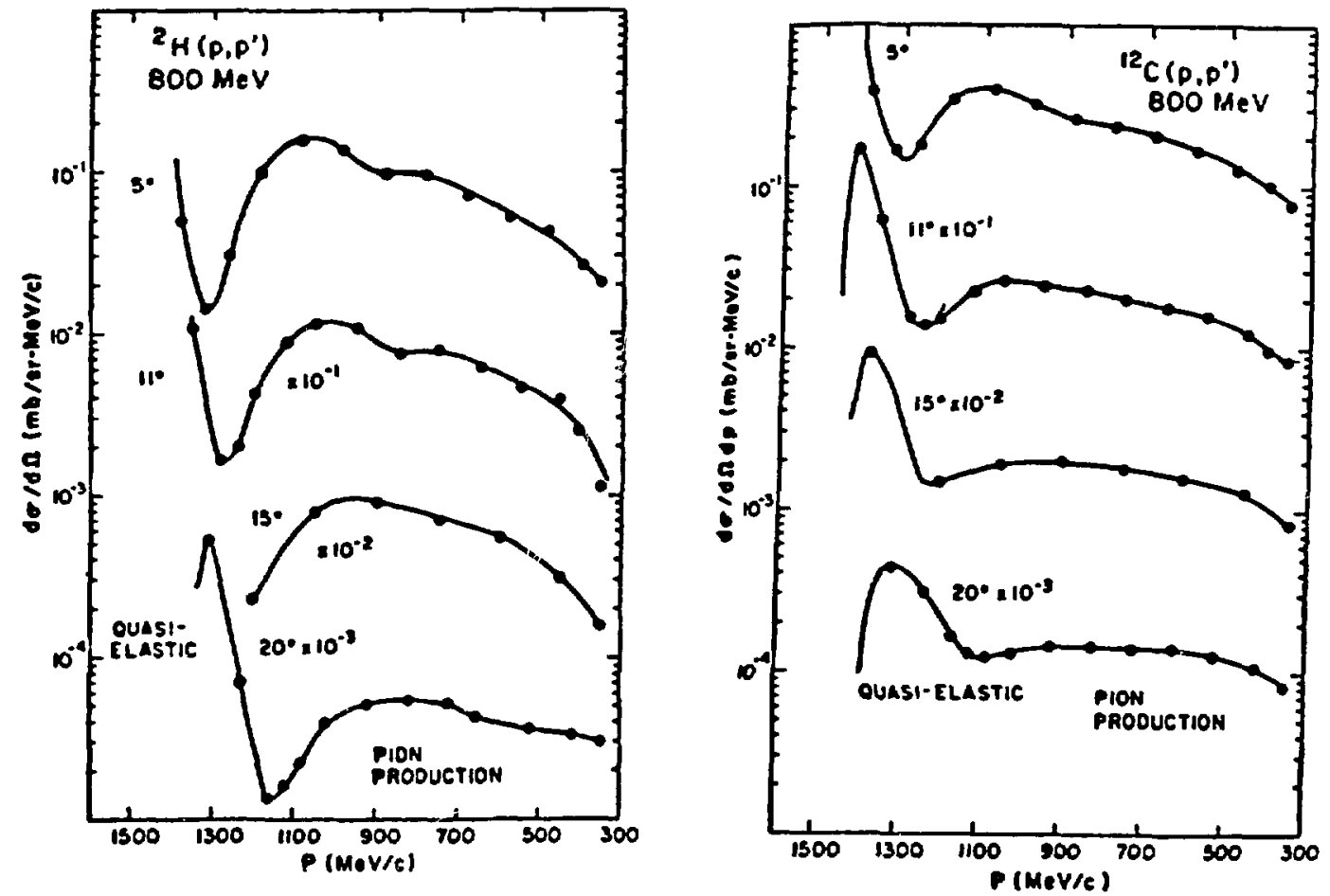

Fig. 24 Inclusive proton scattering spectra for ${ }^{2} \mathrm{H}$ and ${ }^{12} \mathrm{C}$ targets. The beam momentum is $1460 \mathrm{MeV} / \mathrm{c}$. Regions labelled pion production involve quasi-free $\Delta$-production. 
both spectra. The quasi-elastic NN scattering shows up as a sharper peak at smaller energy loss. The proton -12 C reaction cross section inferred from such spectra indicates about one-third is due to quasi-free $\mathrm{NN} \rightarrow \mathrm{NN}$ initiated processes and rwo-thirds is due to quasi-free $N N \rightarrow N \Delta$ initiated processes 123,124 ). A very recent calculation by $\mathrm{Krimm}, \mathrm{Klar}$ and Pirner ${ }^{125}$ ) reports success in describing the ${ }^{12} \mathrm{C}\left(\mathrm{p}, \mathrm{p}^{\prime}\right)$ 'spectra based on using the eikonal approximation and a quasi-free multiple scattering theory related to the one discussed above.

Specific nuclear r-actions can

be chosen which favor the observation of isobars. A recent example is provided by the $\mathrm{p}+{ }^{3} \mathrm{He} \rightarrow \mathrm{x}+{ }^{3} \mathrm{H}$ reaction where $850 \mathrm{MeV}$ protons are incident on ${ }^{3} \mathrm{He}$ and the triton is observed in the final state at forward angles ${ }^{126}$ ). Figure 25 shows the missing mass observed for the partner to ${ }^{3} \mathrm{H}$ and there is a clear enchancement at the $\Delta$-isobar mass.

Fig. 25 Missing mass spectrum for $\mathrm{p}+{ }^{3} \mathrm{He} \rightarrow{ }^{3} \mathrm{H}+\mathrm{x}$ at three triton angles ${ }^{126}$ ).

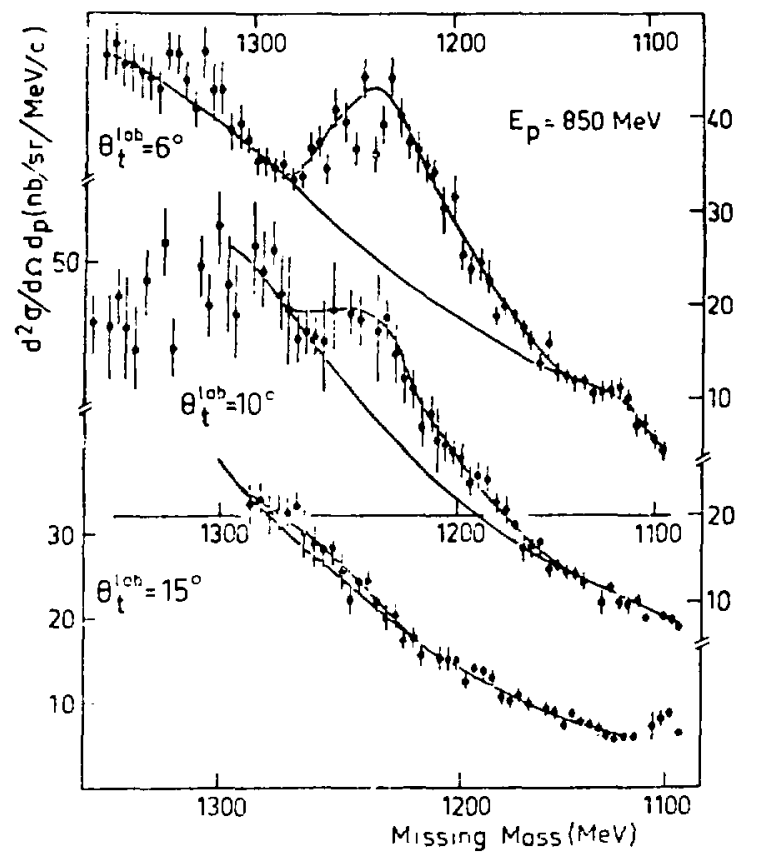

\section{Summary}

Although asymptotic methods of analysis are very old, their application to high energy hadron nucleus diffraction is quite new. The bright spots which have been found to dominate proton-nucleus diffraction at nonforward angles provide both insight and simplification to theoretical analyses as well as a valuable guide to what information can be extracted from proton scattering. The development of phenomenological "data-to-data" relations for inelastic scattering to collective states suggests an underlying simplicity that mainly depends on the asymptotic normalization of the transition density at large $r$. To ext=act more detailed information seems to require the observation of significant differences between the inelastic scattering and the data-to-data predictions based on elastic scattering.

The development of direct connections between simple features of the data and the underlying geometric and dynamical ingredients of multiple diffraction theory is important. It is the basic feature which distinguishes the asymptotic theories from alternative approaches where the contact with the underlying dynamics is not made explicit. It is evident that effective NN amplitude parameters which are able to fit proton-nucleus data can be most easily deduced using the asymptotic theories. However, there are more parameters than can be determined from proton scattering by spin-zero nuclei. More difficult experiments involving the scattering of polarized protons from polarized light ions are required to complete a systematic program of measurements. As has been emphasized, these experiments provide a very fundamental testing ground for proton-nucleus dynamics. A much sharper confrontation between the "free" NN amplitudes and the "effective" amplitudes is likely by the time of the next conference in this series. 
The role of isobars in proton-nucleus interactions begins to become vistble in quasi-free.scattering and in specific nuclear reactions. At present, the role of isobars is under intense investigation in nucleon-nucleon dynamics. Progress in nucleon-nucleon and proton-nucleus research is intertwined in this regard; however, there are clear theoretical reasons for suspecting the isobar intermediate-states of the NN interaction to be modified in the nucleus. A theory of multiple scattering which explicitly displays the isobar production channel has been developed.

Support of the U. S. Department of Energy for this research is gratefully acknowledged.

\section{REFERENCES}

1) G. D. Alkhazov, et al., Phys. Lett. 42B (1972) 121

2) G. D. Alkhazov, et al., JETP Lett. 18 (1973) 181

3) G. D. Alkhazov, et al., Phys. Lett. 57B (1975) 47

4) G. D. Alkhazov, et al., Sov. J. Nucl. Phys. 22 (1975) 469

5) G. D. Alkhazov, et al., Sov. J. Nuc1. Phys. $\frac{26}{5}$ (1977) 357

5) G. D. Alkhazov, et a1., JETP Lett. 26 (1977) 550

7) G. D. Alkhazov, et al., Phys. Lett. 70B (1977) 20

8) G. D. Alkhazov, et al., JETP Lett. 26 (1977) 102

9) G. D. Alkhazov, et al., Sov. J. Nuc1. Phys. 27 (1978) 333

10) G. D. Alkhazov, et al., JETP Lett. 28 (1978) 660

11) G. D. Alkhazov, et al., Joint Institute of Nuclear Research preprint LNPI No. 448, Leningrad (1978)

12) R. Bertini, et al., Phys. Lett. 45B (1973) 119

13) S. D. Baker, et al., Phys. Rev. Lett. 32 (1974) 839

14) G. D. Alkhazov, et a1., Nuc1. Phys. A2 $\overline{74}$ (1976) 443

15) E. Aslanides, Phys. Lett. 68B (1977) 221

16) G. D. Alkhazov, et al., Phys. Lett. 67B (1977) 402

17) G. D. Alkhazov, et a1., Phys. Lett. $\overline{90 B}$ (1980) 364

18) G. S. Blanpied, et al., Phys. Rev. Lett. 39 (1977) 1447

19) G. S. Blanpied, et al., Phys. Rev. C18 (1978) 1436

20) G. W. Hoffmann, et al., Phys. Rev. Lett. 40 (1978) 1256

21) G. W. Hoffmann, et al., Phys. Lett. $76 \mathrm{~B}$ (1978) 383

22) G. W. Hoffmann, et a1., Phys. Lett. $\frac{79 B}{(1978)} 376$

23) J. Fong, et al., Phys. Lett. 78B (1978) 205

24) N. J. DiGiacomo, et al., Phys. Rev. C19 (1979) 1132

25) G. Igo, et a1., Phys. Lett. 81B (1979) 151

26) G. S. Adams, et al., Phys. Rev. Lett. 43 (1979) 421

27) G. Blanpied, et al., Phys. Rev. C20 (1979) 1490

28) L. Ray, et a1., Phys. Rev. C23 (1981) 828

29) G. S. Blanpied, et al., Phys. Rev. C23 (1981) 2599

30) G. S. Kyle, et a1., Phys. Lett. 91B (1980) 353

31 ) D. A. Hutcheon, et a1., Elastic scattering of $400 \mathrm{MeV}$ protons by ${ }^{208} \mathrm{~Pb}$, submitted to Phys. Rev. Lett. (1981)

32) J. P. Auger and R. J. Lombard, Phys. Lett. 45B (1973) 115

33) J. P. Auger and R. J. Lombard, Nuovo Cimento 21A (1974) 529

34) I. Ahmad, Nucl. Phys. A247 (1975) 418

35) R. J. Lombard and C. Wilkin, Lett. al. Nuovo Cimento 13 (1975) 463

36) J. P. Auger, J. Gillespie and R. J. Lombard, Nucl. Phys. A262 (1976) 372

37) R. D. Viollier, Ann. Phys. 93 (1975) 335

38) G. D. Alkhazov, Nucl. Phys. A280 (1977) 330

39) G. K. Varma and L. Zamick, Phys. Rev. C16 (1977) 308; G. K. Varma and

L. Zamick, Nuc1. Phys. A306 (1978) 343

40) D. R. Harrington and G. K. Varma, Nuc1. Phys. A306 (1978) 477

41) J. P. Auger and R. J. Lombard, Ann. Phys. (N.Y.) 115 (1978) 442

42) G. Fäldt and I. Hulthage, J. Phys. G. 4 (1978) 363

43) G. Fäldt and I. Hulthage, Nucl. Phys. $\overline{\mathrm{A} 302}$ (1978) 433

44) J. P. Auger and R. J. Lombard, Nuc1. Phys. A316 (1979) 205

45) G. Fäldt and P. Osland, Nucl. Phys. A305 (1978) 509 
46) P. Osland and R. J. Glauber, Nucl. Phys. A326 (1979) 255

47) R. J. Glauber and P. Osland, Phys. Lett. $80 \mathrm{~B}$ (1979) 401

48) M. Bleszynski and P. Osland, Phys. Lett. 84B (1979) 157

49) Y. Abgra11, J. Labarsouque, B. Morand and E. Caurier, Nucl. Phys. A316 (1979) 389; J. Phys. G. 6 (1980) L55

50) J. P. Auger and R. J. Lombard, Phys. : Lett. 90B (1980) 201

51) S. I. Manaenkov, Sov. J. Nucl. Phys. 31 (1980) 189

52) G. Fäldt and A. Ingemarsson, Uppsala Univ. preprint GWI-PH 2/81 (unpub1ished)

53) G. Fäldt and A. Ingemarsson, Uppsala Univ. preprint GWI-PH 5/81 (unpublished)

54) D. R. Harrington, Nucl. Phys. A343 (1980) 417

55) I. Ahmad and J. P. Auger, Nucl. Phys. A352 (1980) 425

56) R. J. Lombard, G. D. Alkhazov and 0. A. Domchenkov, Nuc1. Phys. A360 (1981) 233

57) E. Kujawski and J. P. Vary, Phys. Rev. C12 (1975) 1271

58) E. Boridy and H. Feshbach, Ann. Phys. (N.Y.) 109 (1977) 468

59) W. R. Coker, L. Ray and G. W. Hoffmann, Phys. Lett. 64B (1976) 403

60) I. Brissaud and M. K. Brusse1, Phys. Rev. C15 (1977) 452

61) L. Ray and W. R. Coker, Phys. Rev. C16 (1977) 340

62) A. Chaumeaux, V. Layly and R. Schaeffer, Phys. Lett. 72B (1977) 33

63) A. Chaumeaux, V. Layly and R. Schaeffer, Ann. Phys. 116 (1978) 247

64) L. Ray, G. W. Hoffmann, G. S. Blanpied, W. R. Coker and R. P. Liljestrand, Phys. Rev. C18 (1978) 1756

65) L. Ray, W. R. Coker and G. W. Hoffmann, Phys. Rev. C18 (1978) 2641

66) L. Ray, Phys. Rev. C19 (1979) 1855; Phys. Rev. C20 (1979) 1212(E)

67) L. Ray, G. S. Blanpied, W. R. Coker, R. P. Liljestrand and G. W. Hoffmann, Phys. Rev. Lett. 40 (1978) 1547

63) C. Gustafsson and E. Lambert, Ann. Phys. 111 (1978) 304

69) L. Ray, et al., Phys. Lett. 83B (1979) $27 \overline{5}$

70) L. Say, G. S. Blanpied and W. R. Coker, Phys. Rev. C20 (1979) 1236

71) L. Ray and P. E. Hodgson, Phys. Rev. C20 (1979) 2403

72) L. Ray, Phys. Rev. C20 (1979) 1857

73) G. D. Alkhazov, S. L. Belostotsky and A. A. Vorobyov, Phys. Rep. 42C (1978) 89

74) C. A. Whitten, Jr., in High energy physics and nuclear structure, eds. D. F. Measday and A. W. Thomas, Nucl. Phys. A335 (1980) 419

75) L. Ray in High energy physics and nuciear structure, eds. D. F. Measday and A. W. Thomas, Nuc1. Phys. A335 (1980) 443

76) G. J. Igo, Proc. of Santa Fe Conference on polarization phenomena (1980)

77) A. W. Thomas in Proc. of the Int. Conf. on nuclear physics, eds. R. M. Diamond and J. O. Rasmussen, Nuc1. Phys. A354 (1981) 51c.

78) S. J. Wallace in Advances in nuclear physics, vol. 12 (Plenum, New York, 1981) p. 135

79) R. D. Amado, J.-P. Dedonder and F. Lenz, Phys. Rev. C21 (1980) 647

80) R. J. Glauber and M. Bleszynski, Asymptotic theory of diffractive scattering, contribution to this conference; M. Bleszynski, R. J. Glauber and P. Osland, Harvard University preprint (1981)

81) R. G. Newton, Scattering theory of waves and particles (McGraw-Hi11, New York, 1966) p. 77, 94

82) J. F. Germond and M. Johnson, Phys. Rev. C22 (1980) 1622

83) W. Frahn, University of Paris-Sud preprint IPNO/TH 80-44 (1980)

84) E. V. Inopin and Y. A. Berezhnoy, Nuc1. Phys. 63 (1965) 689

85) T. E. 0. Ericsson, in Proc. Int. Conf. on nuclear structure, J. Phys. Soc. of Japan 44 (supplement) (1978)

86) R. J. Glauber, in Lectures in theoretical physics, eds. W. E. Brittin and

L. G. Dunham, vol. 1 (Interscience, New York, 1959) p. 315

87) J. W. Negele and D. Vautherin, Phys. Rev. C5 (1972) 1472

88) R. D. Amado, J. A. McNeil and D. A. Sparrow, Phys. Rev. C23 (1981) 2114

89) This value is cited as $0.16+10.20 \mathrm{fm}$ in ref. 87 , however there is an apparent sign reversal since the polarization in NN scattering must be positive in eq. (26).

90) R. D. Amado, F. Lenz, J. A. McNeil and D. A. Sparrow, Phys. Rev. C22 (1980) 2094; J. A. McNeil and D. A. Sparrow, Phys. Rev. C23 (1981) 2124 
91) R. D. Amado, J. A. McNeil and D. A. Sparrow, Phys. Rev. C23 (1981) 2186

92) L. J. Tassie, Aust. J. Phys. 9 (1956) 407

93) H. Spinka, in Proc. of Workshop on nuclear and particle physics and energies up to $31 \mathrm{GeV}$, eds. J. D. Bowman, L. S. Klsslinger and R. R. Silbar, Los Alamos publication LA-8775-C (Los Alamos Scientific Laboratory, 1981) p. 220

94) W. M. Kloet and R. R. Silbar, Nucl. Phys. A338 (1980) 281, 1bid.317; Phys. Rev. Lett. 45 (1980) 970

95) W. Kloet, J.A. Tjon and R. R. Silbar, Phys. Lett. 99B (1981) 80

96) C. L. Hollas, Phys. Rev. Lett. 44 (1980) 1186

97) D. V. Bugg, et a1., Phys. Rev. $\overline{\mathrm{C} 21}$ (1980) 1004; D. V. Bugg, et al., J. Phys. G 4 (1980) 1025

98) R. Arndt, private communication of phase shift solution CD79 (unpublished)

99) N. Hoshizaki, Prog. Theor. Phys. (Japan) 60 (1978) 1796

100) W. G. Love and M. A. Franey, Univ. of Minnesota preprint (1981)

101) W. Grein and P. Kroll, Nucl. Phys. 137B (1978) 173

102) A. Wreikat, et al., Phys. Lett. 97B (1980) 33; F. Irom, G. J. Igo, J. B. McClelland and C. A. Whitten, Jr., submitted to Phys. Rev. (1981)

103) S. J. Wallace and Y. Alexander, Phys. Lett. 90B (1980) 346

104) J. A. McNeil, I: Relativistic eikonal exfansion. II: Proton-deuteron scattering at intermediate energies, University of Maryland Ph.D. Dissertation (1979), unpublished

105) M. Bleszynski, et al., Phys. Lett. 87B (1979) 198

106) G. Alberi, M. Bleszynski, T. Jaroszewicz and S. Santos, Phys. Lett. 92B (1980) 41

107) J. V. Geaga, et al., Phys. Rev. Lett. 38 (1977) 1265

108) H. Courant, et al., Phys. Rev. C19 (1979) 104

109) B. J. VerWest, private communication

110) A. M. Green, in Reports on progress in physics, ed. J. M. Ziman, vol. 39 (Institute of Physics, London, 1979) p. 1109

111) A. M. Green, in Mesons in nuclei, eds. M. Rho and D. H. Wilkinson, vol, 1 (North-Holland, Amsterdam, 1979) p. 227

112) M. Betz and T.-S. H. Lee, Phys. Rev. C23 (1981) 375

113) E. Abers, H. Burkhardt, V. L. Teplitz and C. Wilkin, Nuovo Cimento A42 (1966) 365

114) J. Pumplin and M. Ross, Phys. Rev. Lett. 21 (1968) 1778

115) J. S. Trefil, Nucl. Phys. 34B (1971) 109; J. S. Trefil, Phys. Rev. D3 (1971) 1615

116) M. Ikeda, Phys, Rev. C6 (1972) 1608

117) S. A. Gurvitz, R. D. Amado and J.-P. Dedonder, Phys. Rev. C19 (1979) 142

118) J. P. Auger, C. Lazard and R. J. Lombard, University of Paris-Sud preprint IPNO-TH 81-21 (1981)

119) L. Kisslinger and W. Wang, Ann. Phys. (N.Y.) 99 (1976) 374

120) M. Hirata, J. H. Koch, F. Lenz and E. $\mathcal{~ . ~ M o n i z , ~ A n n . ~ P h y s . ~ ( N . Y . ) ~} 120$ (1979) 205

121) S. J. Wallace, V. B. Mandelzweig, D. M. Schneider and J. W. Van Orden, to be published, Phys. Rev. C (1981)

122) R. E. Chrien, et al., Phys. Rev. C21 (1980) 1014

123) G. W. Hof fmann, private communication

124) Y. Alexander, J. W. Van Orden, E. F. Redish and S. J. Wallace, Phys. Rev. Lett. 44 (1980) 1579

125) H. Krim, A. Klar and H. J. Firner, University of Heidelberg preprint UNI-HD-81-1 (1981) unpublished

126) B. Tatischeff, et ai., University of Paris-Sud preprint IPNO-PhN-78-09 (1981) unpublished 